\title{
Anabases
}

ANABASES Traditions et réceptions de l'Antiquité

26 | 2017

Varia

\section{In situ et alibi, dallo scavo integrato alla cultura dell'abitare: Vesuviana a Ercolano}

Antonella Coralini

\section{(2) OpenEdition}

Journals

Edizione digitale

URL: http://journals.openedition.org/anabases/6274

DOI: 10.4000/anabases.6274

ISSN: 2256-9421

Editore

E.R.A.S.M.E.

\section{Edizione cartacea}

Data di pubblicazione: 1 novembre 2017

Paginazione: 67-102

ISSN: 1774-4296

\section{Notizia bibliografica digitale}

Antonella Coralini, «In situ et alibi, dallo scavo integrato alla cultura dell'abitare: Vesuviana a Ercolano»,

Anabases [Online], 26 | 2017, Messo online il 01 novembre 2020, consultato il 20 janvier 2021. URL:

http://journals.openedition.org/anabases/6274 ; DOI: https://doi.org/10.4000/anabases.6274

(c) Anabases 


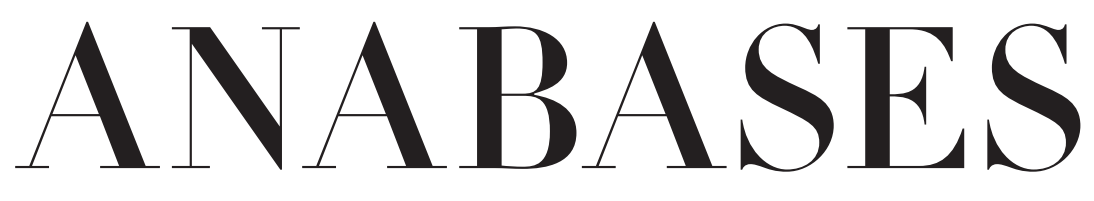

Traditions et Réceptions de l'Antiquité

$$
\begin{aligned}
& N \circ 26 \\
& 2017
\end{aligned}
$$

\section{E.R.A.S.M.E.}

Université Toulouse - Jean Jaurès 



\section{Sommaire}

$\mathrm{N}^{\circ} 26-2017$

\section{Historiographie et identités culturelles}

Jocelyne Peigney

La polysémie poétique d'anabase chez Saint-John Perse :

évocations, étymologie et botanique $\ldots \ldots \ldots \ldots \ldots \ldots \ldots \ldots \ldots \ldots$

Nicolas FAELLI

Les références antiques des fondateurs de l'Amérique française

au XVII siècle ................................. I9

Silvia Alaura

Setting the stage for Hittite studies in Victorian England:

practices and methods of the 1870 . . . . . . . . . . . . .

\section{Traditions du patrimoine antique}

“ Restituer Herculanum I. Des archives de fouilles aux restitutions 3D »

Alexandra Dardenay et Adeline Grand-Clément

Restituer Herculanum au XXI ${ }^{\mathrm{e}}$ siècle. L'apport des archives. . . . . . . . 55

Antonella Coralini

In situ e alibi, dallo scavo integrato alla cultura dell'abitare :

Vesuviana-DHER a Ercolano . . . . . . . . . . . . . . . . 67 67

Agnes Allroggen-Bedel

I documenti settecenteschi come strumenti per lo studio

degli scavi ercolanesi . . . . . . . . . . . . . . . Io3

James ANDREws

Revisiting the upper floors of the casa di Argo at Herculaneum . . . . . . II7 
Nicolas MonteIx

Les archives des fouilles d'Herculanum au XX $\mathrm{XX}^{\mathrm{e}}$ siècle :

analyse critique et pistes d'exploitation . . . . . . . . . . I43

\section{Archéologie des savoirs}

Yannick Le PAPE

L'inspiration et l'imprudence. Poésie de l'anticomanie

dans la critique d'art du second XIX ${ }^{\mathrm{e}}$ siècle . . . . . . . . . . . . . I57

\section{Actualités et débats}

Claude AzızA

Antiquités parallèles (7). La starlette des bandelettes . . . . . . . . . I77

Alexandra TrachseL

La réception d'Homère dans le domaine de la rhétorique $\ldots \ldots \ldots$. . . I83

Claude Aziza

Curiosa (2) In vino (vin/eau) veritas (very tasse) : drame de l'ivresse . . . . I89

\section{Lire, relire la bibliothèque des sciences de l'Antiquité}

Laurent OLIVIER

Pour en finir avec la race : Henri Hubert et l'anthroposociologie

À propos du commentaire de “L'Aryen, son rôle social », de Vacher de Lapouge $(\mathrm{r} 899) \ldots \ldots \ldots$

Henri Hubert

Compte rendu de Georges VACher de LAPouge, L'Aryen, son rôle social, L'Année sociologique, igoo, p. i45-146 . . . . . . . . . . . . . . . . . 203

\section{L'atelier de l'histoire : chantiers historiographiques}

L'Atelier des doctorants (coordonné par Adeline Grand-Clément) (14)

Francesco LoDÀ,

L'initiation des guerriers chez les peuples indo-européens.

Une enquête historiographique dans les pas de Georges Dumézil . . . 207

\section{Comptes rendus}

Benjamin Anderson et Robert G. Ousterhout

Palmyra I885. The Wolfe Expedition and the photographs

of John Henry Haynes (M. Sartre) . . . . . . . . . . . . . . . . . . . 2 215 
Mary R. BAchvarova

From Hittite to Homer. The Anatolian Background of Ancient Greek Epic (G. Hoffmann) . . . . . . . . . . . . . . . . .

Patrick Baker, Ronny Kaiser, Maike Priesterjahn, Johannes Helmrath (éd.) Portraying the Prince in the Renaissance. The Humanist Depiction of Rulers in Historiographical and Biographical Texts (G. Demelemestre). . 220

Brigitte BAKHоUсHE (éd.)

Science et exégèse. Les interprétations antiques et médiévales du récit biblique de la création des éléments (Genèse I, I-8) (F. P. BARONE) . . . . . . .

Laurence Baurain-Rebillard (éd.)

Héros grecs à travers le temps : autour de Persée, Thésée, Cadmos et Bellérophon (C. Giovénal) . . . . . . . . . . . . . . . .

Mark W. PAdiLla,

Classical myth in four films of Alfred Hitchcock . . . . . . . . . . . . . . .

Vivien Bessieres

Le Péplum et après? L’Antiquité gréco-romaine dans les récits contemporains (Cl. Aziza) . . . . . . . . . . . . . . . . . 225

Pierre Briant

Alexandre. Exégèse des lieux communs (É. Guillon) . . . . . . . . . . . . . 227

Paulo ButTi DE Lima

Il piacere delle Immagini. Un tema aristotelico nella riflessione moderna sull'arte (C. Noacco) . . . . . . . . . . . . . . . . . . .

Bénédicte Coudì̀re

La truelle et le phylactère. La proximité des images (M. Scapin) . . . . . . . 23I

Thorsten Fögen, Richard WARREN (éd.)

Graeco-Roman Antiquity and the Idea of Nationalism in the rgth Century, Case Studies (M. Champier) . . . . . . . . . . . . . . . ${ }_{232}$

Ronald H. Fritze

Egyptomania. A History of Fascination, Obsession and Fantasy

(Fabien Bièvre-Perrin) . . . . . . . . . . . . . . . . . . . . . 234

Joseph Geiger

Hellenism in the East. Studies on Greek Intellectuals in Palestine

(C. Bonnet). . . . . . . . . . . . . . . . . 236

Tiffany Jenkins

Keeping their marbles. How the treasures of the past ended up in museums... and why they should stay there (C. Valenti) . . . . . . . . . . 238 
Ayelet H. Lushkov

Magistracy and the Historiography of the Roman Republic.

Politics in Prose (C. Landrea) . . . . . . . . . . . . . . . . . . . . . . 240

Jean-Claude MüHLEthaler

Énée le mal-aimé. Du roman médiéval à la bande dessinée

(Fl. Bouchet) ........................ 24I

Laurent PERNoT

Epideictic Rhetoric, Questioning the Stakes of Ancient Praise

(C.Psilakis) ......................... 243

Priscian

Answers to King Khosroes of Persia (O. Gengler) . . . . . . . . . . . . . . 245

Paul Robertson

Paul's letters and contemporary Greco-Roman literature:

theorizing a new taxonomy (M. Cambron-Goulet) . . . . . . . . . . . . . . 247

Federico Santangelo (éd.)

Approaching the Roman Revolution. Sir Ronald Syme,

Papers on Republican History (C. Landrea) . . . . . . . . . . . . . . . . . . 249

Molly Swetnam-BurLand

Egypt in Italy. Visions of Egypt in Roman Imperial Culture

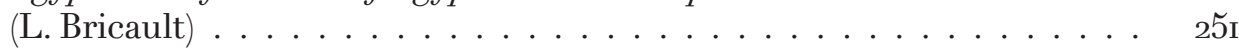

Helen VAN Noorden

Playing Hesiod. The 'Myth of the Races' in Classical Antiquity

(D. Bonanno)

Résumés . . . . . . . . . . . . . . . . . . . . . . . . . . . . . . . 257

Index .......................... . . 265 


\section{Traditions}

\section{du patrimoine antique}

Restituer Herculanum I.

Des archives de fouilles aux restitutions 3D 



\title{
In situ et alibi, dallo scavo integrato alla cultura dell'abitare: Vesuviana a Ercolano
}

\author{
Antonella Coralini
}

\section{Dagli archivi storici agli archivi digitali}

Come per ogni sito dalla lunga storia conservativa, oggetto di una serie discontinua e disomogenea di interventi di recupero e musealizzazione, anche per gli Scavi di Ercolano, ora Parco, una componente fondamentale del percorso di conoscenza e valorizzazione è costituita dall'archeologia degli archivi e dei depositi, ovvero dalla riscoperta e dalla rilettura "stratigrafica» della documentazione prodotta nel corso di quella lunga storia e dei reperti tuttora individuabili ${ }^{\text {. }}$

I risultati di questi scavi alibi possono essere utilmente organizzati in nuovi archivi, digitali, mettendo a frutto le potenzialità dell'archeologia virtuale, ed in particolare la possibilità di fare dei modelli tridimensionali l'interfaccia per la gestione dei risultati della ricerca, dai dati primari ai metadati e paradati².

1 Sull'archeologia degli archivi e dei depositi, ovvero sugli scavi alibi, linea di azione di fondamentale importanza nei progetti del programma Vesuviana dell'Università di Bologna, A. Coralini, “Vesuviana (1997-). Lavorare per progetti», in A. Coralini (a cura di), Vesuviana. Archeologie a confronto, Atti del Convegno Internazionale (Bologna, 14-16 gennaio 2008), Bologna, 2009, pp. 41-56; EAD., “DHER - Domus Herculanensis Rationes (2005-)», ibidem, pp. 41-56; EAD., "Ercolano Resina Herculaneum», in A. Coralini (a cura di), DHER. Domus Herculanensis Rationes. Sito archivio museo, Bologna, 2011, in partic. pp. 11-46.

2 Sul tema, per il caso di studio pompeiano, A. Coralini, E. Vecchietti, “L’archeologia attraverso un 3D Virtual Model», in A. Coralini, D. Scagliarini (a cura di), Ut natura ars. Archeologia e Virtual Reality, Atti del Convegno Internazionale (Bologna, 21 
Tutte le tappe di questo percorso sono state state sperimentate nel progetto ercolanese dell'Università di Bologna, per il tramite di specifiche linee di azione ed attività, che hanno fatto dell'antica Ercolano il caso di studio per un progetto di ricerca di documentalistica applicata all'archeologia, dal recupero della documentazione storica alla realizzazione di nuove campagne di documentazione dell'evidenza materiale tuttora esistente, dal trattamento dei dati così acquisiti alla loro comunicazione ${ }^{3}$.

Di questa sperimentazione ultradecennale, avviata nei primi anni Duemila, le pagine che seguono propongono una sintesi, per tracciare bilanci e delineare prospettive.

Due sono stati e sono i fils rouges di questo percorso: la vocazione ad una lettura sempre contestuale, dal livello fisico a quelli immateriali, dall'antico al moderno e contemporaneo, e l'obiettivo ultimo, contribuire ad una migliore conoscenza della cultura abitativa di Ercolano, ma sulla base di una versione rivista, aggiornata ed arricchita della base documentaria, su doppia scala, di città e di singole unità, edilizie ed urbanistiche ${ }^{4}$.

\section{I contesti di un progetto, fra realtà fisica e contingenze immateriali}

A vent'anni dalla sua nascita, Vesuviana, il programma quadro creato nel 1997 dall’Università di Bologna al fine di mettere a sistema il suo contributo alla migliore conoscenza e alla valorizzazione dei siti vesuviani, continua ancor oggi, nel 2017, a coltivare la sua vocazione per un approccio integrato e transdisciplinare al suo

aprile 2002), Bologna 2007, pp. 17-40; per il campione ercolanese, A. Coralini et alii, "ViSMan-DHER: a Virtual Archaeology Application for Visualization and Management of Archaeological Data Related to ancient Herculaneum (NA), Italy", in VAST 2010. 11th International Symposium on Virtual reality, Archaeology, and Cultural Heritage (Paris, 21-24/09/2010), pp. 71-74, Goslar, 2010; per una riflessione di metodo, A. Coralini, "Antichi vicini di casa. Presenze reali e virtuali nel mondo digitale», in J. Bonetto, M. Salvadori (a cura di), Atti del Convegno, L'architettura privata ad Aquileia in età romana (Padova, 21-22 febbraio 2011), Padova 2012, pp. 639664.

3 Coralini, 2011, con bibliografia precedente, fra cui un saggio di ampio respiro e di fondamentale importanza: M. Ferraris, Documentalità. Perché è necessario lasciar tracce, Roma-Bari, 2009.

4 L'obiettivo è condiviso, e perseguito con strategie e modalità complementari, anche dall'Herculaneum Conservation Project, sin dal 2000, e dal più recente progetto VESUVIA condotto dall'Università di Toulouse (A. Dardenay, H. Eristov, “Herculanum, habitat et société. Analyse des appareils architecturaux et décoratifs pour une approche socio-historique de la cité antique», Chronique des activités archéologiques de l'École française de Rome, Roma, 2013). 
oggetto di studio, preso in esame in tutte le sue componenti, fra antico, moderno e contemporaneo, grazie alla virtuosa convergenza di più discipline ${ }^{5}$.

Dopo aver concentrato attenzione ed energie, nei suoi primi anni di vita, sul campione pompeiano, con il suo progetto pilota, "Pompei. Insula del Centenario (IX 8)" (1998-), il programma Vesuviana ha esteso, sin dall'inizio degli anni Duemila, la sua sfera di interesse e il suo ambito di azione al campione ercolanese, sia per ampliare gli orizzonti della sua lettura contestuale, sia per testare l'applicabilità del modello di intervento messo a punto sul campione pompeiano.

I primi passi in questa direzione sono stati compiuti sin dal 2000, attraverso alcune verifiche di fattibilità, anche nella forma di progetti di ricerca individuali, dedicati, in particolare, all'analisi del repertorio figurato della pittura parietale e alla definizione dello status quaestionis per l'insula III, in vista della sua selezione come caso di studio 6 .

Risale al 2005 l'avvio in forma ufficiale del nuovo progetto, Domus Herculanensis Rationes (DHER), finalizzato allo studio, alla valorizzazione e alla comunicazione della cultura abitativa dell'antica Ercolano, attraverso due principali linee di azione, a scala di sito e a scala di insula, fra loro complementari: l'Atlante degli Apparati Decorativi di Ercolano e l'analisi di dettaglio dell'insula III.

Nel nuovo scenario, quello del sito deuteragonista, in rapporto a Pompei, fra i siti vesuviani, il programma Vesuviana ha conservato e potenziato le linee-guida delle sue scelte di metodo e del suo modus operandi, dall'archeologia degli archivi e dei depositi (gli scavi alibi) alla realizzazione di nuove e sistematiche campagne di rilievo e documentazione dell'evidenza materiale, in situ e in Museo, fra approccio transdisciplinare, con una forte attenzione per l'archeometria del costruito e della produzione e per l'archeologia virtuale, solida vocazione all'integrazione sinergica fra ricerca e didattica, che costituisce il quid di molti progetti di ricerca di matrice universitaria e che ne fa dei percorsi di lunga durata, potenzialmente sine die, in un processo di sperimentazione, e formazione, aperto e continuo.

Anche nel progetto ercolanese, come già in quello pompeiano, gli scavi in archivio e in deposito hanno preceduto le attività in situ, quali componente fondamentale della necessaria verifica di fattibilità del progetto stesso, per poi affiancarsi ad esse.

5 Vesuviana, 2009, con bibliografia precedente; A. Coralini, "Vesuviana". L'Alma Mater a Ercolano, Pompei, Napoli. Attività e risultati dal 2006 al 2013, in Rivista di Studi Pompeiani 24 (2013), p. 117-123, A. Coralini, “Vesuviana (2001-2016). Spazi e decorazioni, fra città e territorio", in Rivista di Studi Pompeiani 26-27 (2015-2016), p. 164-172.

6 A. Coralini, “La pittura parietale di Ercolano. I temi figurati», Ocnus, 13 (2005), p. 169-198. 
Teatri principali degli scavi alibi sono stati, oltre agli archivi e ai depositi della Soprintendenza, ed in particolare degli Scavi di Ercolano, l'Archivio di Stato di Napoli e i giacimenti di dati e materiali del Museo Archeologico Nazionale di Napoli, realtà di primaria importanza per ogni ricerca sui siti vesuviani.

Un ruolo fondamentale è stato svolto, sin dalla fase preliminare, di verifica di fattibilità, e sino al 2016, dalla stretta collaborazione, fondata su specifiche convenzioni di ricerca, con gli enti per la tutela (il Museo Archeologico Nazionale di Napoli e la Soprintendenza Autonoma di Pompei) e, per il tramite della Direzione dell’Ufficio Scavi di Ercolano, nella persona di Maria Paola Guidobaldi, con l'Herculaneum Conservation Project ${ }^{7}$.

Il risultato è stato una feconda rete di rapporti di condivisione e scambio di competenze e dati, che ha coinvolto tutti gli attori impegnati nello scenario ercolanese e che nel progetto DHER ha visto affiancarsi all'équipe di archeologi quelle di specialisti di altri settori disciplinari. Dal 2005 ad oggi al progetto hanno infatti collaborato esperti di geomatica e di scienza delle costruzioni, quali i colleghi del DISTART, ora DAAM, dello stesso Ateneo bolognese, oltre a chimici specializzati in archeometria per i beni culturali, da più Università (Bari, Modena e Reggio Emilia, Parma, Venezia), nell'ambito di un programma di ricerca di rilevante interesse nazionale (PRIN, 2005-2007), e a tecnologi delle applicazioni ICT ai beni culturali, con il Vis.I.T. Lab del CINECA ${ }^{8}$. Più di recente (dal 2016) la rete di collaborazioni si è arricchita dell'ingresso nel partenariato dell'Università di Napoli L'Orientale, attraverso il suo Centro Interdipartimentale di Servizi di Archeologia (CISA), per il rilievo e documentazione, e dell'Università IUAV di Venezia, per la diagnostica e lo studio del degrado?.

Oltre che in interim reports, presentazioni a convegni e contributi in sedi specializzate, il programma di lavoro e i progressi delle attività hanno trovato un adeguato esito editoriale nel primo volume di studi, opera collettiva coordinata

7 Sull'Herculaneum Conservation Project, in generale, la sezione ad esso dedicata in Vesuviana, 2009, p. 197-290, e, per le specifiche attività, gli interim reports pubblicati annualmente nel Notiziario della Rivista di Studi Pompeiani, quale, ad esempio, D. Camardo, M. Notomista, Indagine archeologica nel braccio est del peristilio della Casa dell'Albergo, in RivStPomp 21 (2010), pp. 152-153.

8 Per i risultati di questo approccio transdisciplinare, l'opera collettiva realizzata nell'occasione del primo lustro di vita del progetto, DHER, 2011. E' ora in corso di preparazione il secondo volume, dedicato all'insula III, con focus sul rapporto fra spazi costruiti e spazi decorati, fra interventi antichi e moderni (Spazi e decorazioni: l'insula III).

9 Grazie alla collaborazione dei colleghi Andrea D'Andrea, per l'Università di Napoli L'Orientale (CISA), e, per l'Università IUAV di Venezia, Emanuela Sorbo e Fabrizio Antonelli. 
e diretta dalla scrivente, DHER. Domus Herculanensis Rationes, nel 2011, alla scadenza del primo lustro di vita ufficiale del progetto, e ad un decennio dall'inizio delle verifiche di fattibilità.

Raggiunti e superati i suoi quindici anni di vita effettiva, il progetto ercolanese di Vesuviana dà continuità al suo impegno per la migliore conoscenza e la valorizzazione dell'antica Ercolano, con il medesimo obiettivo: indagarne la cultura abitativa, analizzata in tutti i suoi aspetti e restituita per più vie, dalla documentazione e ricostruzione dei suoi apparati decorativi, parietali e pavimentali in primis, alla modellazione tridimensionale e virtuale dei suoi edifici, fra assetto moderno, frutto dei restauri voluti da A. Maiuri, e assetto antico, così come ipotizzabile sulla base dei dati offerti da un approccio integrato.

E' su questo obiettivo e su questi risultati che si realizza la virtuosa convergenza delle due linee di azione principali del progetto DHER, lo studio di dettaglio dell'insula III e l'Atlante degli Apparati Decorativi. Alla loro intersezione si colloca il lavoro sull'insula III, oggetto del secondo volume di studi, di prossima pubblicazione (DHER. Spazi e decorazioni: l'insula III).

Per realizzare le finalità del progetto ercolanese, che prevedono lo studio contestuale sia degli apparati decorativi, sia, in prospettiva più ampia, delle forme e dei modi dell'abitare (così come dichiara anche il suo titolo, Domus Herculanensis Rationes), Vesuviana ha scelto di attivare due linee di ricerca e di intervento sul terreno, unendo ad un approccio trasversale, "a scala di città), approfondimenti “in verticale», a scala di edificio e di insula.

La prima linea si propone la documentazione e l'analisi degli apparati decorativi strutturali, in situ et alibi, cioè sia di quanto conservato nel sito, sia delle parti asportate e musealizzate altrove. Il risultato atteso è l'Atlante degli Apparati Decorativi di Ercolano, un prodotto fotogrammetrico e georeferenziato, concepito come strumento di lavoro aperto, cioè accessibile a tutta la comunità scientifica, per il tramite dell'ente per la tutela, con cui i dati vengono condivisi in itinere.

La seconda prevede lo studio di dettaglio di unità abitative, ad iniziare da quelle appartenenti all'insula che è stata selezionata d'intesa con la Direzione degli Scavi come caso campione del progetto DHER, l'insula III, ed è finalizzata all'edizione scientifica di singoli complessi.

Alla base di entrambe sta lo stesso metodo di lavoro, che fonda il suo approccio interdisciplinare su nuove e sistematiche campagne di acquisizione dei dati primari, per più vie: rilievo e documentazione dell'evidenza materiale (strutture, decorazioni, reperti mobili); verifica dei dati d'archivio; recupero e studio dei materiali dei vecchi scavi, e quindi rilettura critica della vulgata. 


\section{A scala di sito: I'Atlante degli Apparati Decorativi di Ercolano}

Paradigma per ironia della sorte, anche nella storia degli studi sulla pittura parietale romana i siti archeologici vesuviani hanno svolto e continuano ad avere il ruolo di termine di riferimento obbligato, in forza della consistenza quantitativa della loro evidenza materiale e del buono (o comunque superiore alla media) stato di conservazione degli affreschi da essi restituiti.

Ne è derivata una sorta di assioma dell'integrità degli edifici di Pompei e Ercolano, solo di recente superato dalla consapevolezza di come i due siti solo in apparenza conservino sistemi decorativi parietali leggibili in modo completo e di come in realtà siano invece il risultato di una lunga serie di interventi moderni finalizzati alla ricomposizione e alla conservazione. La secolare storia dell'indagine archeologica e delle scelte di restauro e di presentazione ha fatto delle pitture vesuviane un insieme particolarmente complesso, da affrontare con approccio duttile e modulabile di caso in caso.

Sin dal suo avvio, il programma quadro Vesuviana ha scelto di fondare l'analisi e l'interpretazione dell'esistente su una solida base documentaria, realizzata $e x$ novo e ad hoc.

In questo percorso di ricerca e formazione una tappa fondamentale corrisponde alla creazione, presso il Dipartimento di Storia Culture e Civiltà, del Laboratorio di Rilievo e restituzione della pittura antica (2005-): un esperimento di successo che è riuscito nell'intento di coniugare ricerca e alta formazione e dove sono anche gli archeologi stessi ad acquisire le competenze tecniche necessarie per la documentazione e la ricostruzione degli apparati decorativi antichi.

Due sono stati i suoi principali scenari di azione, affrontati con approcci diversificati, calibrati sulle specificità del campione: da una parte, Pompei e la Casa del Centenario (IX 8, 3. 6, a), i cui apparati decorativi, a quasi 140 anni dalla scoperta, risultano compromessi in modo più o meno grave; Ercolano, dall'altra, dove maggiore è la difficoltà di ricostruire le vicende più antiche dello scavo in cavo cieco, attraverso i pozzi e i cunicoli della stagione borbonica, e di riconoscere l'antico al di là e al di sotto degli interventi conservativi succedutisi sin dai primi recuperi e soprattutto del piano di ricomposizione e restauro voluto da A. Maiuri in seguito allo scavo a cielo aperto e alla creazione della sua "città-museo"10.

Per quanto riguarda la prima linea di azione del progetto DHER, quella dell'Atlante, la ricca letteratura scientifica relativa all'antica Ercolano, nonostante i contributi significativi che negli ultimi decenni si sono affiancati a quella che ne

10 Sul tema, poi sviluppato nella recente monografia di E. Sorbo, Tra materia e memorie. Ercolano (1711-1761), Savignano sul Rubicone 2014, già V. Papaccio, A. Salvatori, “Ercolano: stratificazione degli interventi ed esigenza del restauro documentale», in Le Scienze, le istituzioni, gli operatori alla soglia degli anni 90', Atti del convegno di Studi (Bressanone, 21-24 giugno 1988), Padova 1988, pp. 195a-205a. 
resta tuttora l'opera maior, I nuovi scavi di Ercolano di A. Maiuri (1958) ${ }^{11}$, continua ad essere più che evidente un'ampia lacuna nelle ricerche e negli studi.

A mancare è soprattutto un'opera equivalente a quello che per il principale fra i siti vesuviani, Pompei, è costituito dall'impresa, prima documentale e poi editoriale, che ha prodotto i quattro volumi di Pitture e Pavimenti di Pompei (1981-1992) e di "Pompei. Pitture e mosaici" (1990-1998) e dal progetto Haueser in Pompeji ${ }^{12}$. Il corredo iconografico su cui fondare studi di sintesi o analisi di dettaglio consiste, per Ercolano, solo in una documentazione d'archivio realizzata non per scopi di ricerca scientifica, ma per esigenze legate alla gestione del sito, alla quale si aggiungono i risultati di campagne di rilievo finalizzate a specifici progetti editoriali (quali le edizioni scientifiche della Casa dell'Atrio a Mosaico, IV, 2,1 e della Casa del Colonnato Tuscanico) ${ }^{13}$.

L'esito è una documentazione discontinua e disomogenea, alla quale il progetto DHER, con il suo Atlante degli Apparati Decorativi, vuole affiancare un nuovo

1 Fra questi, dopo i contributi di R.E.L.B. De Kind, Houses in Herculaneum: A New View on the Town Planning and the Building of Insulae III and IV, Circumvesuviana, 1, Amsterdam, 1998 е Тн. Ganschow, Untersuchungen zur Baugeschichte in Herculaneum, Bonn, 1989, si segnalano soprattutto D. Esposito, La pittura parietale di Ercolano, Roma, 2014; F. Guidobaldi, M. Grandi, M.S. Pisapia, Mosaici antichi in Italia. Regione prima. Ercolano, Pisa-Roma 2014; G. Losansky, Die Obergeschossareale der Stadthäuser in Herculaneum, Reichert, 2015, per il problema dei piani superiori (tema preso in esame anche nella Ph.D, dissertation di J. N. Andrews, The use and development of upper floors in houses at Herculaneum, 1-3, Thesis submitted for the Degree of Ph.D., Department of Archaeology, University of Reading 2006) e D. Camardo, M. Notomista (a cura di), Ercolano: 1927-1961. L'impresa archeologica di Amedeo Maiuri e l'esperimento della città museo, Studi e ricerche del Parco archeologico di Pompei, 1, Roma, 2017.

12 Pompei. Pitture e Pavimenti, I-III, Roma, 1981-1986 ; Pompei. Pitture e mosaici, I-IX, Roma, 1990-1998; Haueser in Pompeji, I-XII (F. Zevi, V.M. Strocka, Case di Pompei. Un programma di documentazione archeologica, Napoli, 1980; V.M. SтrockA, W. Ehrhardt, “Case di Pompei: un progetto scientifico internazionale dell'Istituto Archeologico Germanico", in Rivista di Studi Pompeiani 1 (1987), p. 203-208). Per l'impostazione di metodo, I. BaLdassarre, “Lo studio delle pitture pompeiane oggi», in P.G. Guzzo (a cura di) Pompei. Scienza e società. 250 anniversario degli Scavi di Pompei Atti del Convegno Internazionale, (Napoli, 25-27 novembre 1998), Milano, 2001, p. 137-138.

13 M.G. Cerulli Irelli, Le pitture della Casa dell'atrio a mosaico, Monumenti della pittura antica scoperti in Italia, 3. La pitture ellenistico-romana, Ercolano, 1, Roma, 1971; EAD., La Casa del Colonnato tuscanico ad Ercolano, Memorie dell'Accademia di Archeologia, Lettere e Belle arti di Napoli, 7, Napoli, 1974; M. Manni, Ercolano, 2. Le pitture della Casa del colonnato tuscanico, Roma 1974. 
strumento di lavoro, condiviso e verificabile, a due livelli: il primo, indispensabile, consiste in un completo atlante fotogrammetrico, esteso all'evidenza in situ e a quella alibi, mentre il secondo è costituito, come passo successivo e complementare, dal corredo, edificio per edificio, delle restituzioni grafiche delle superfici decorate e delle relative schede di catalogo (in entrambi i casi realizzate sulla base dei fotopiani e delle ortofoto dell'atlante fotogrammetrico, rilette anche alla luce dei dati desumibili dall'esame della documentazione fotografica d'archivio e di verifiche autoptiche mirate).

L'obiettivo è quindi quello di realizzare per Ercolano una completa base documentaria, indispensabile per lo studio d'insieme della cultura abitativa, anche nell'ottica della conservazione e della comunicazione al pubblico, e quindi per l'analisi degli apparati decorativi e lo studio contestuale degli stessi, sia nel sistema abitativo sia nel contesto più ampio della città.

Per entrambi i livelli, di rilievo fotogrammetrico e di restituzione grafica, il lavoro, avviato nel 2005, è oggi giunto ad un buono stadio di avanzamento, nonostante i limiti oggettivi creati dalle esigenze di gestione del sito, che hanno reso sino ad ora non accessibile un non piccolo numero di complessi.

Per il primo decennio di attività sono tredici le unità edilizie per le quali è stato possibile completare il rilievo fotogrammetrico, dall'acquisizione dei dati alla loro rielaborazione ${ }^{14}$, e per otto di queste si è già proceduto anche alle due azioni successive, di restituzione grafica e catalogo (fig. 1) ${ }^{15}$.

Per la linea di ricerca "a scala di città", quella dedicata alla lettura contestuale degli apparati decorativi strutturali di Ercolano, sono stati quindi raggiunti due importanti risultati. Da una parte, è stato possibile acquisire la documentazione relativa ad alcuni dei più raffinati complessi residenziali della città, quali le Case del Tramezzo di Legno (III, 11), dei Cervi (IV, 21) e del Salone Nero (VI, 11-13). Dall'altra, è stato inoltre preso in esame un buon numero di edifici minori, che costituiscono comunque un campione di rilevante interesse, come documenti dell'ultima fase decorativa della città, preziosi soprattutto per lo studio delle trasformazioni dello spazio domestico e della messa a punto di nuove forme dell'abitare.

Alla base della linea di azione dell'Atlante, trasversale e a scala di sito, sta una nuova campagna sistematica di documentazione, che fa tesoro delle

14 Case dell'Albergo (III, 1. 19), annessa alla Casa dell'Albergo (III, 18), dello Scheletro (III, 3), del Tramezzo di Legno (III, 4. 11), dell'Erma di Bronzo (III, 16), dell'Ara Laterizia (III, 17), dell'Alcova (IV, 4), di Nettuno e Anfitrite (IV, 7), del Papiro Dipinto (IV, 9), dei Cervi (IV, 21), dell'Atrio Corinzio (V, 30), del Gran Portale (V, 35), del Salone Nero (VI, 13).

15 Case dell'Albergo (III, 1. 19), annessa alla Casa dell'Albergo (III, 18), dello Scheletro (III, 3), del Tramezzo di Legno (III, 4. 11), dell'Erma di Bronzo (III, 16), dell'Ara Laterizia (III, 17), dell'Atrio Corinzio (V, 30), del Salone Nero (VI, 13). 


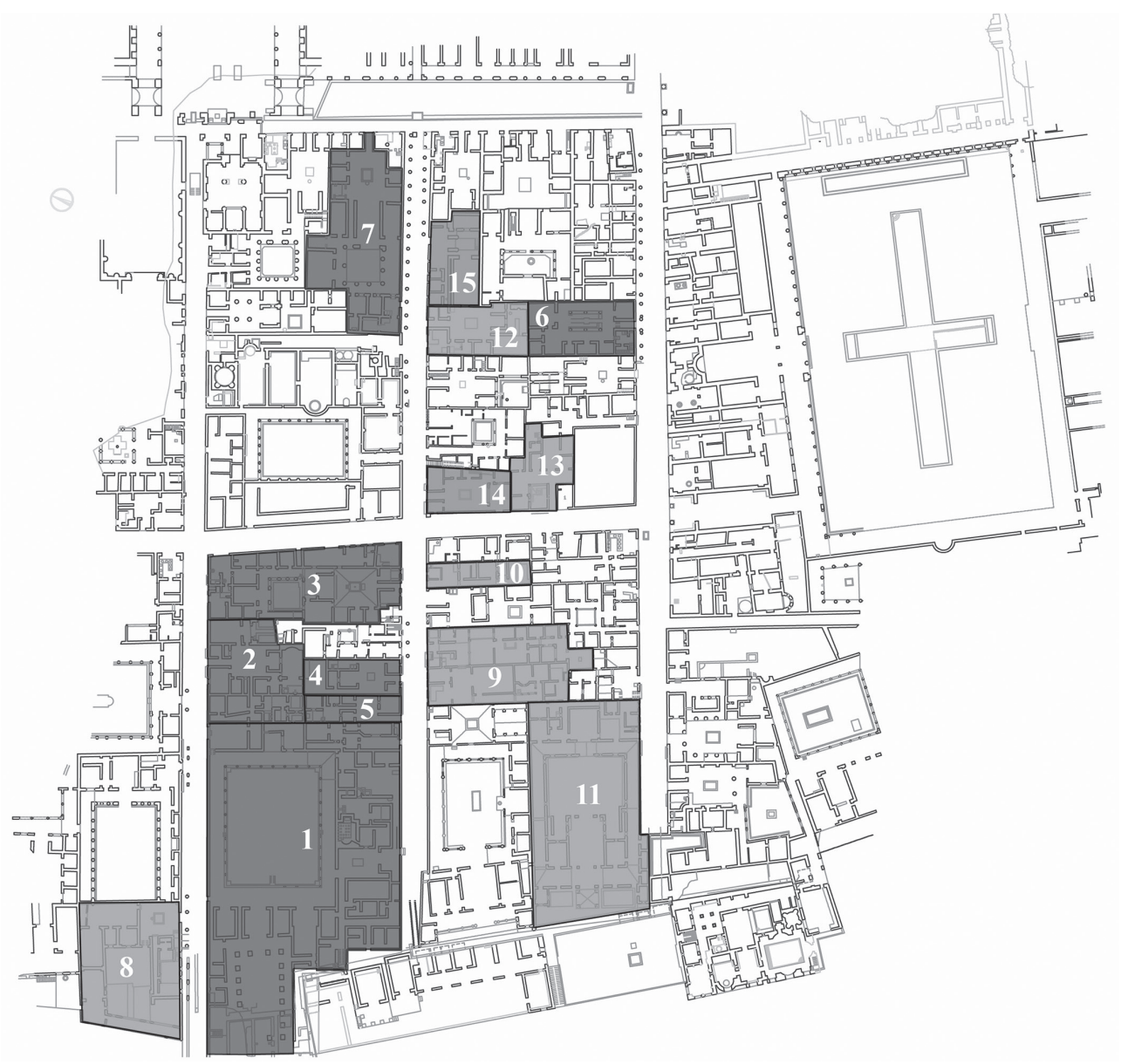

Fig. 1. Ercolano. Progetto DHER, Atlante degli Apparati Decorativi (2005-2016): stato di avanzamento delle attività di rilievo e di restituzione grafica. In grigio scuro, le unità per le quali sono già state realizzate, oltre al rilievo fotogrammetrico, anche la restituzione grafica, lineare e in scala di grigi, e la catalogazione delle superfici decorate.

esperienze maturate nella pluriennale attività nel cantiere-laboratorio dell'Insula del Centenario (IX 8) a Pompei e che applica tecniche e procedure in costante aggiornamento ${ }^{16}$.

16 Per il metodo di lavoro, sulla base delle riflessioni di A. Barbet, “Déontologie de la restitution idéale d'un décor', in Virtual Retrospect 2007, Actes du Colloque (Pessac, 2007) Bordeaux 2008, pp. 269-278; D. Scagliarini, A. Coralini, “Alla ricerca della pittura perduta: metodologie di rilievo per le pareti dipinte», in D. ScagLiarini, A. Coralini, R. Helg, Davvero! La Pompei di fine '800 nella pittura di Luigi Bazzani, 


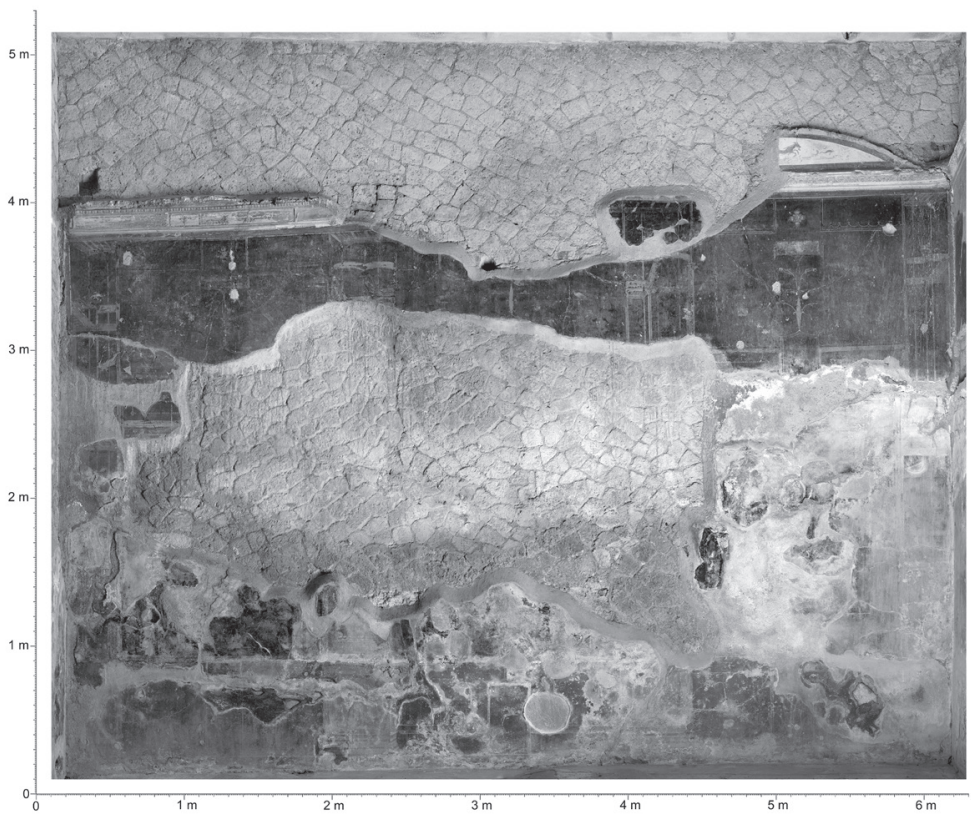

Fig. 2. a. Ercolano, Casa dell'Atrio Corinzio (V, 30), ambiente 2, parete nord: fotopiano, (Programma Vesuviana, DHER, Pascucci, 2008).

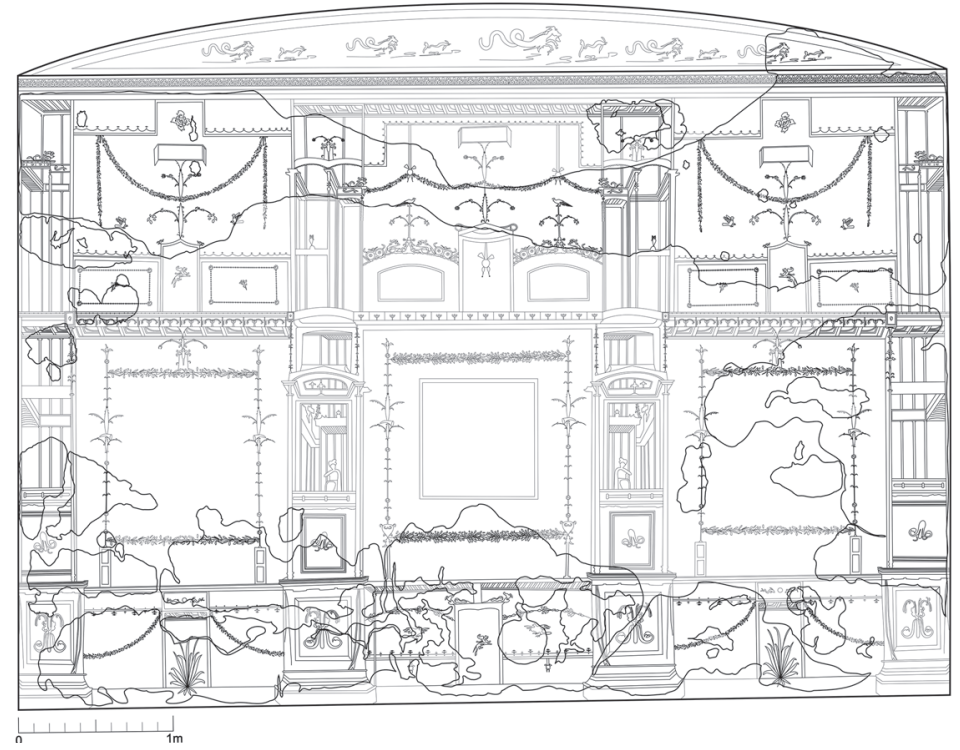

Fig. 2. b. Ercolano, Casa dell'Atrio Corinzio (V, 30), ambiente 2, parete nord: restituzione grafica lineare, (Programma Vesuviana, DHER, V. Bollini, 2011). 


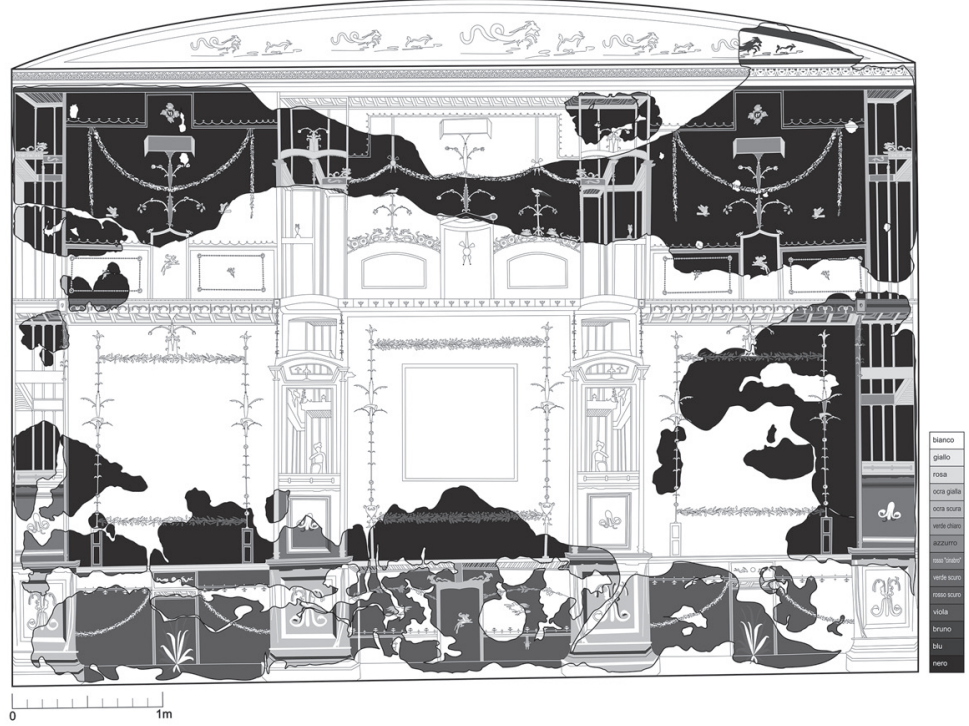

Fig. 2. c. Ercolano, Casa dell'Atrio Corinzio (V, 30), ambiente 2, parete nord: restituzione grafica in scala di grigi, (Programma Vesuviana, DHER, V. Bollini, 2011).

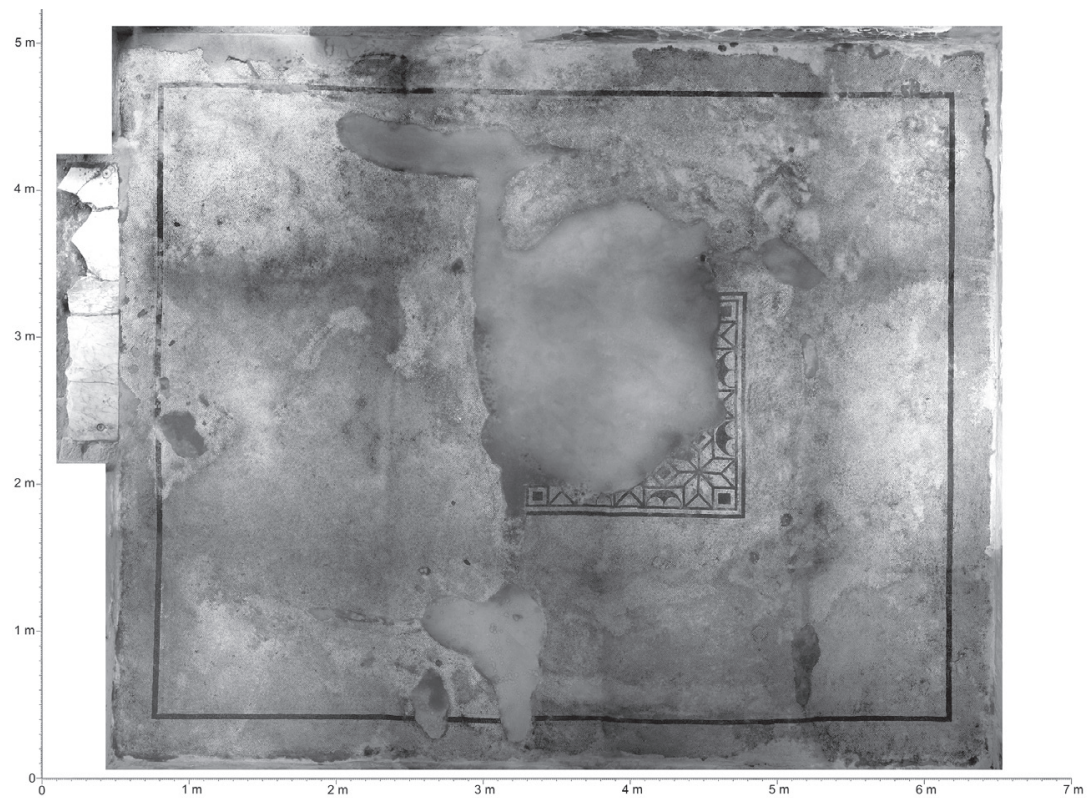

Fig. 2. d. Ercolano, Casa dell'Atrio Corinzio (V, 30), ambiente 2, parete nord: pavimento, fotopiano (Programma Vesuviana, DHER, C. Pascucci, 2008). 
Nella fase di acquisizione dei dati, il rilievo fotogrammetrico dell'evidenza materiale, in situ et alibi, fornisce l'indispensabile base di lavoro alle due azioni complementari, cioè all'analisi autoptica (che ricorre all'esame in luce radente della superficie pittorica, alla ricerca di indicatori delle tecniche di esecuzione e delle modalità di organizzazione del lavoro dei pictores) e alla restituzione grafica integrativa delle decorazioni.

L'osservazione delle pitture in luce radente, oltre che in luce naturale, può infatti consentire di acquisire numerose informazioni sui modi di realizzazione, e in particolare sulle soluzioni (schizzi preparatori; linee guida incise o tracciate a pennello sull'ultimo strato di intonaco; utilizzo di riga o compasso) adottate per adeguare schemi e motivi alle superfici a disposizione e per far fronte ai tempi stretti imposti dalla tecnica ad affresco.

I dati ricavati dalla verifica autoptica delle pitture in situ, relativi sia alle caratteristiche stilistiche ed esecutive delle pitture, sia allo stato di conservazione delle stesse, vengono registrati in un database relazionale, che permette di ottenere report differenziati e di organizzare i dati in tabelle.

Tali dati vanno ad arricchire ulteriormente, accanto a quelli già forniti dagli ortofotopiani metricamente corretti, la base documentale sulla quale si fonda la restituzione grafica delle decorazioni, che prevede due modalità di rilievo integrativo: lineare (con il rilievo vettoriale delle superfici pittoriche) e in scala di grigi (fig. 2).

Per l'esecuzione di questi prodotti di secondo livello si è fatto ricorso all'utilizzo di uno specifico software di grafica vettoriale open source, Inkscape, scelto non solo in ragione del suo essere open, ma anche per la sua duttilità d'uso: il software consente infatti sia di disegnare in modo diretto su immagini di diversa tipologia, sia di modificare e correggere il disegno in momenti diversi e di modificare le dimensioni e la scala del prodotto finale. Importante, in questo percorso ricostruttivo, è il contributo dì diversi tipi di fonti grafiche e fotografiche, d'insieme e di dettaglio, che anche nel caso di Ercolano si rivelano utili per la ricostruzione della sintassi decorativa delle pareti, laddove testimoniano la presenza di ornati oggi irrimediabilmente perduti a causa del degrado o a seguito della pratica dell'asportazione (di quadri, vignette e motivi figurati), che ha caratterizzato le indagini sette e ottocentesche (figg. 4-5) ${ }^{17}$.

Catalogo della mostra (Bologna, 29 marzo-26 maggio 2013; Napoli, 4 luglio 2013-6 gennaio 2014), Bologna, 2013, p. 69-74.

17 Sulle modalità di trattamento delle pitture dagli scavi nei siti vesuviani, dal Settecento in poi, dopo il pionieristico lavoro di M.P. Rossignani, "Saggio sui restauri settecenteschi ai dipinti di Ercolano e Pompei», in Contributi dell'Istituto di Archeologia, Pubblicazioni dell'Università Cattolica del Sacro Cuore, Milano, 1 (1967), p. 7-134; P. D'Alconzo, Picturae excisae. Conservazione e restauro dei dipinti 


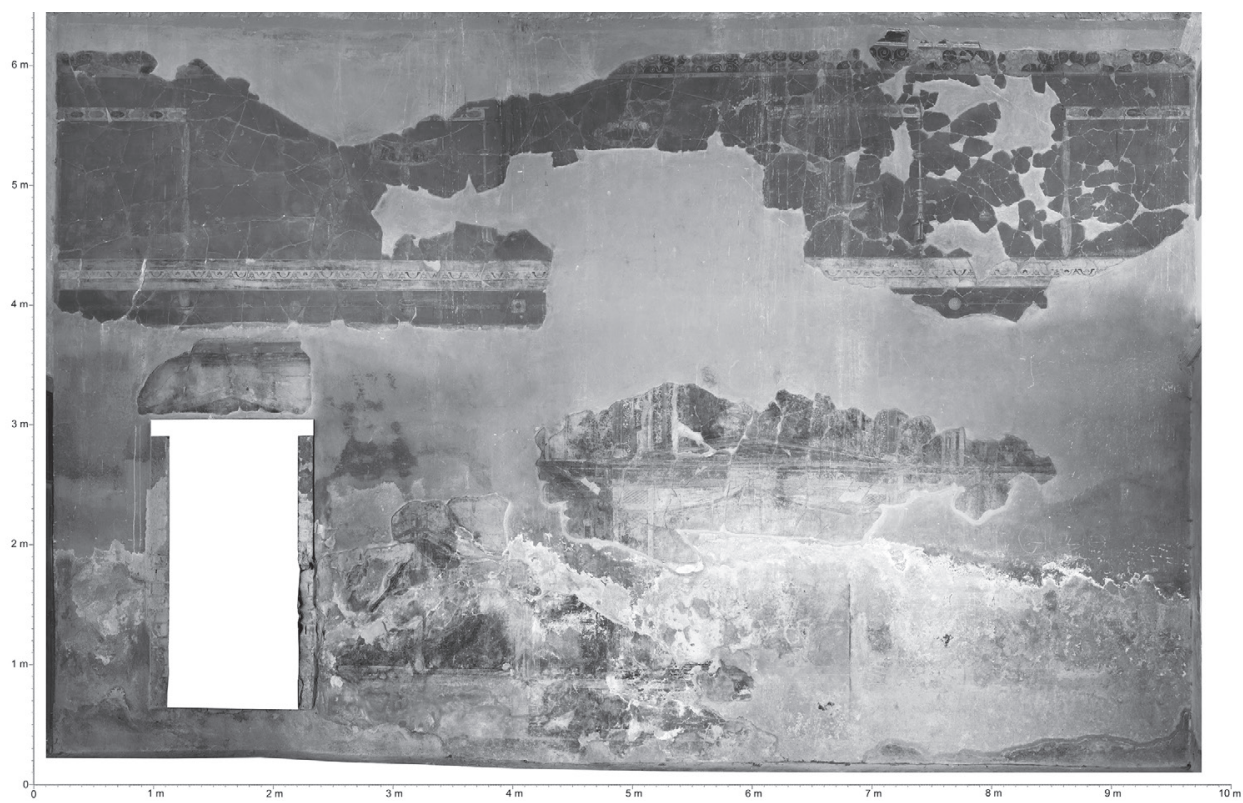

Fig. 3. a. Ercolano, Casa del Tramezzo di Legno (III, 4-12), atrio 20, parete nord: rilievo fotogrammetrico 1:20 (Programma Vesuviana, DHER, a. C. Pascucci, 2011).

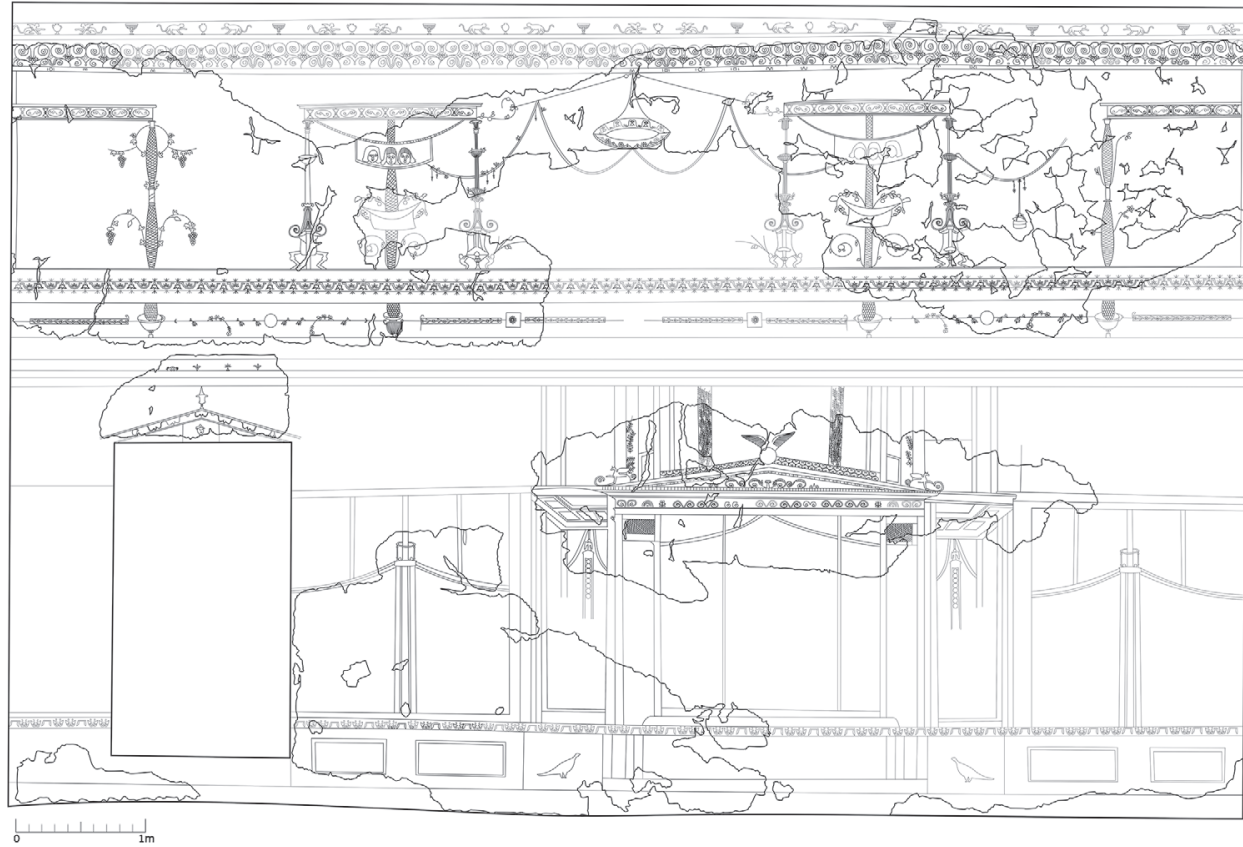

Fig. 3. b. Ercolano, Casa del Tramezzo di Legno (III, 4-12), atrio 20, parete nord: rilievo integrativo lineare (Programma Vesuviana, DHER, L. Galvagni, 2012). 


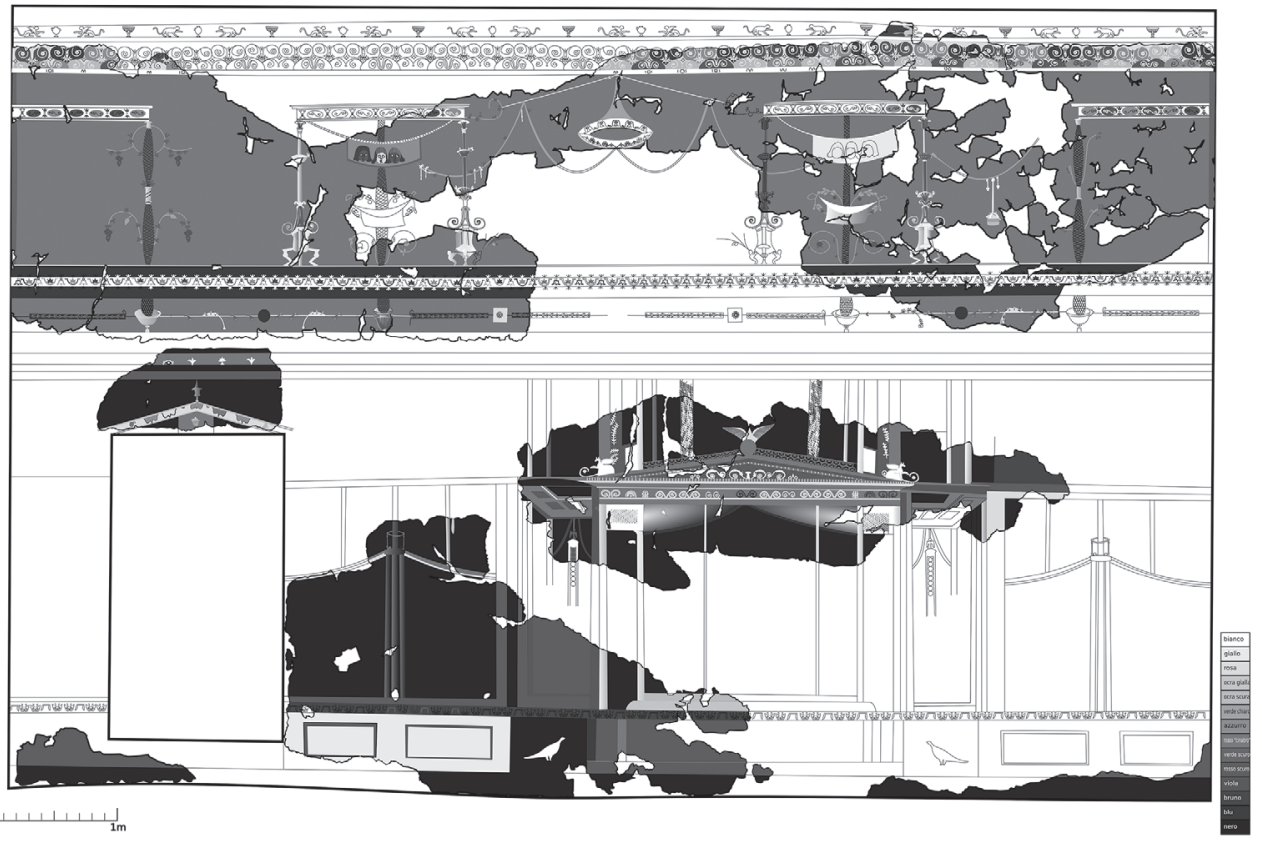

Fig. 3. c. Ercolano, Casa del Tramezzo di Legno (III, 4-12), atrio 20, parete nord: rilievo integrativo in scala di grigi (Programma Vesuviana, DHER, L. Galvagni, 2012).

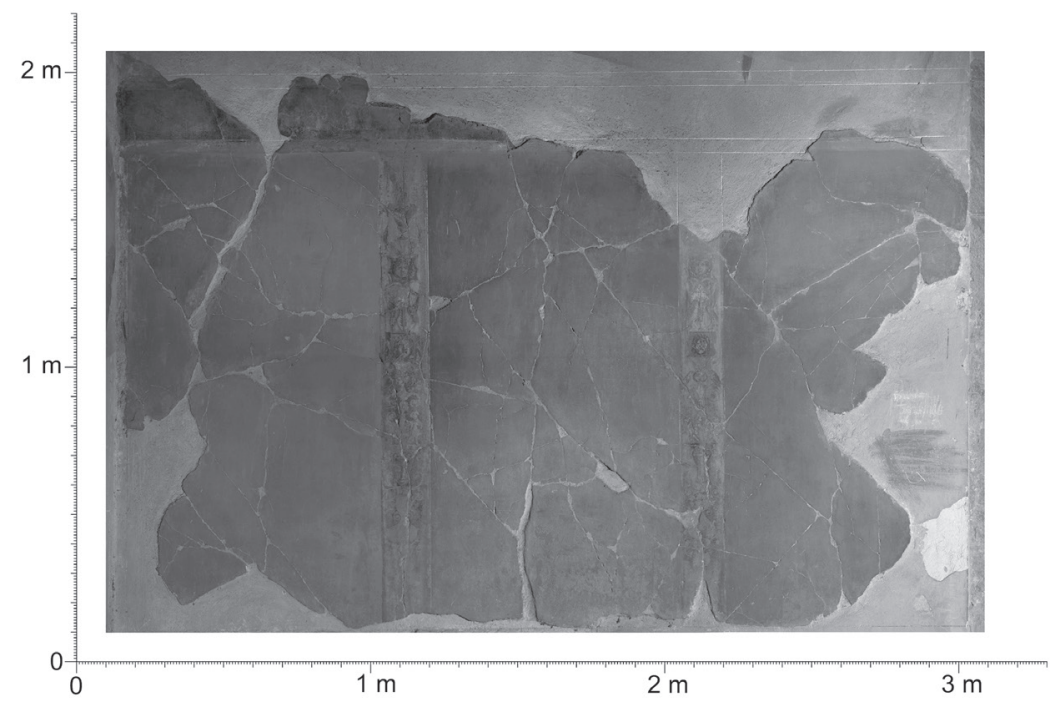

Fig. 4. a. Ercolano, Casa del Tramezzo di Legno (III, 4-12), ala 5, parete ovest: rilievo fotogrammetrico 1:20 (Programma Vesuviana, DHER, C. Pascucci, 2011). 

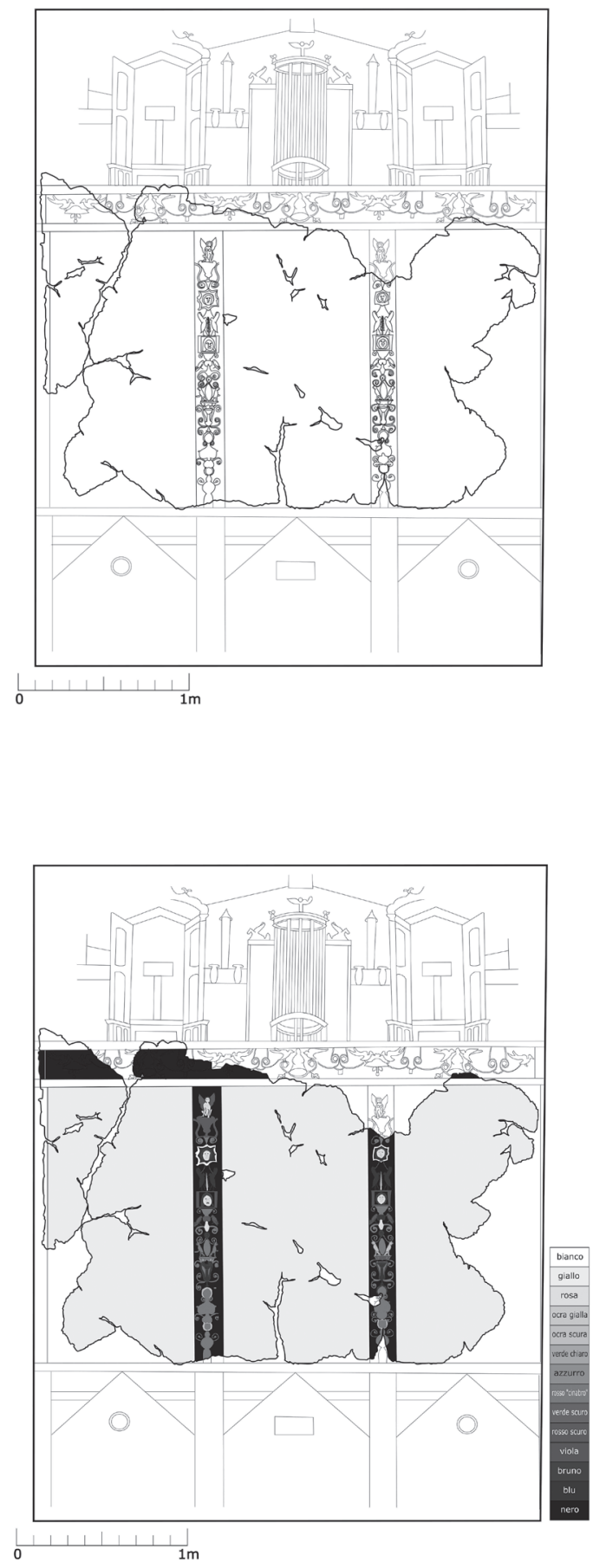

Fig. 4. b. Ercolano, Casa del Tramezzo di Legno (III, 4-12), ala 5, parete ovest: rilievo integrativo al tratto e in scala di grigi (Programma Vesuviana, DHER, L. Galvagni, C. Cordoni, 2013-2015).

Fig. 4. c. Ercolano, Casa del Tramezzo di Legno (III 4-12), ala 5, parete ovest: rilievo integrativo al tratto e in scala di grigi (Programma Vesuviana, DHER, L. Galvagni, C. Cordoni, 2013-2015). 

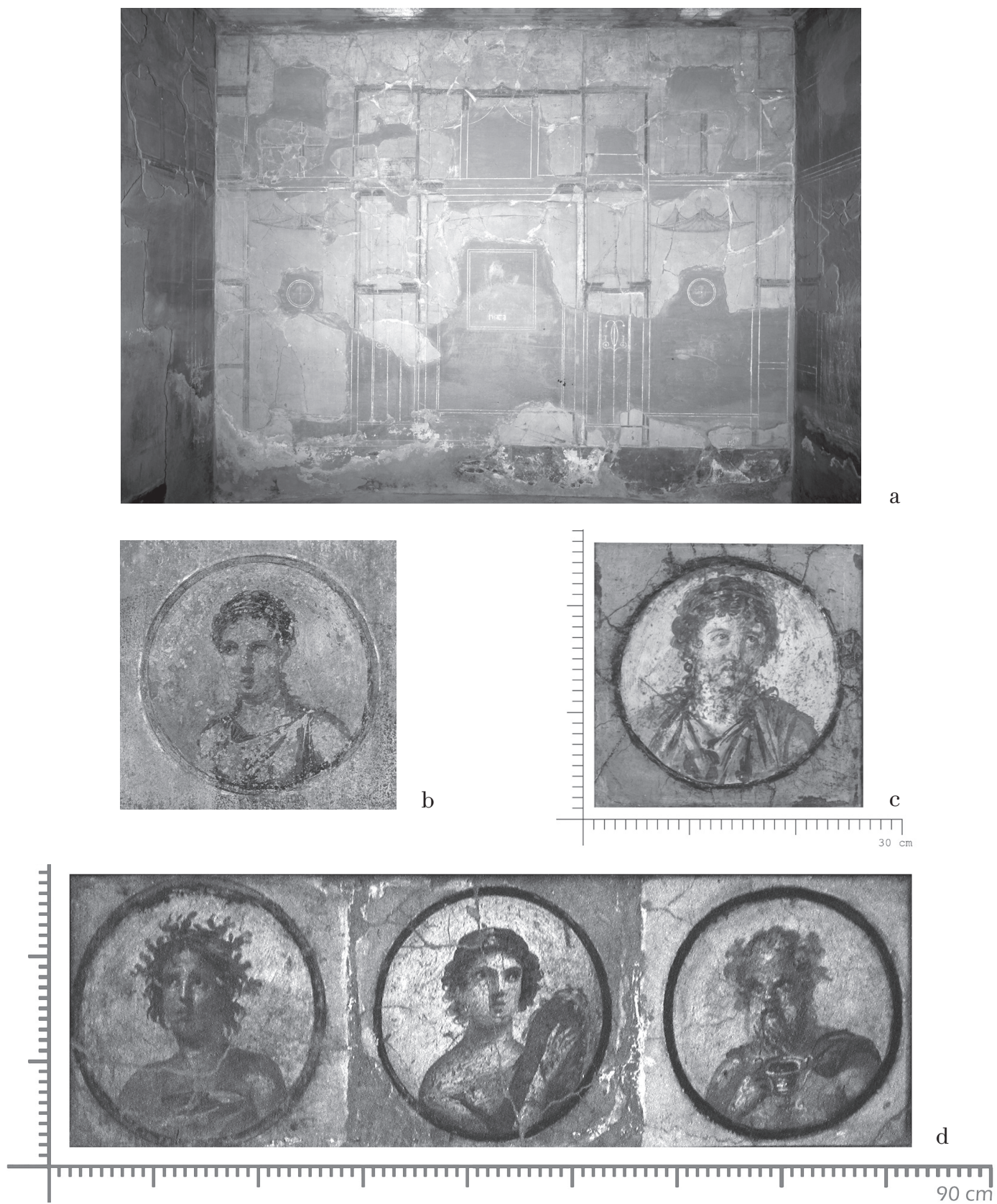

Fig. 5. a-c. Ercolano, Casa dell'Atrio a Mosaico (IV, 1-2), esedra 9. Sulla base delle affinità con il medaglione tuttora in situ (b) e con quelli conservati nei depositi del Museo Archeologico Nazionale di Napoli (d, inv. 9129), il quadro senza numero di inventario n. 156 (c) del Museo risulta attribuibile alla decorazione dell'esedra 9 (a). 
Le potenzialità del software si sono rivelate anche nella resa, indiretta, del cromatismo originale della parete, con l'applicazione alle linee ricostruttive e alle campiture della superficie di una gamma in scala di grigi emulativa della gamma cromatica e tale da consentire una prima percezione del sistema decorativo, secondo una soluzione già sperimentata con successo nell'apparato grafico del progetto Haueser in Pompeji.

Le ricostruzioni così ottenute hanno il valore di prezioso strumento conoscitivo e interpretativo. L'analisi dettagliata dell'evidenza materiale, condotta secondo livelli di approfondimento progressivo, consente infatti di proporre una lettura il più possibile integrata degli apparati decorativi in esame e di valorizzare il potenziale (per la ricerca, la formazione e la comunicazione) che il rilievo e la restituzione grafica possiedono, mettendo a punto metodi e tecniche di acquisizione e rielaborazione dei dati applicabili all'intero sito.

Al lavoro condotto su quanto ancora si conserva in situ ed è restituibile sulla base dell'approccio integrato che unisce alla documentazione storica i risultati di nuove campagne di acquisizione dei dati, strumentali e autoptiche, si è sin dall'inizio affiancata una linea di azione complementare, che ha avuto ed ha come teatro il Museo Archeologico Nazionale di Napoli, nel quadro di un progetto specifico, “Alibi. Pitture in Museo».

Sui disiecta membra del Museo è infatti tuttora in corso, accanto a periodiche campagne di rilievo fotogrammetrico, finalizzate alla realizzazione di un corpus di immagini metricamente corrette complementare a quello delle decorazioni in situ, la verifica sistematica e capillare delle provenienze, condotta in primis sui reperti che nell'Inventario Generale appaiono attribuiti al sito di Ercolano e poi estesa all'intera raccolta, attraverso l'esame delle catene inventariali abbinato a quello formale e tecnico ${ }^{18}$. Fra i risultati più recenti, si segnala il catalogo di un gruppo cospicuo (219 unità) di picturae excisae tuttora non inserite nell'Inventario corrente del Museo, ma in genere presenti in quelli precedenti e perlopiù riconducibili ai primi anni degli scavi nei siti vesuviani. Ciascuno di questi reperti ha cosi acquisito una propria "carta di identità), che consente di valorizzarne il potenziale, sia sul piano della ricerca sia ai fini di una migliore valorizzazione. Per alcuni di essi, inoltre, è stato anche già possibile individuare non solo il sito

ercolanesi e pompeiani tra XVIII e XIX secolo, Studi della Soprintendenza archeologica di Pompei, 8, Roma, 2002; G. Prisco (a cura di), Filologia dei materiali e trasmissione al futuro. Indagini e schedatura sui dipinti murali del Museo Archeologico Nazionale di Napoli, Roma, 2009.

18 Per le verifiche inventariali, per il metodo di lavoro, V. SAmpaolo, «Strumenti inventariali per il riordino della Collezione degli Affreschi del Museo Archeologico Nazionale di Napoli», in Ocnus 13 (2005), p. 283-290; per un esempio applicativo, V. ToмeI, “La natura morta», in DHER, 2011, p. 217-242. 
di provenienza, ma anche lo spazio fisico e l'insieme decorativo di pertinenza: è questo il caso di un medaglione con busto femminile su fondo azzurro, per il quale è stata confermata, sulla base anche dell'affinità formale con quanto si conserva tuttora in situ, l'appartenenza alla decorazione della Casa dell'Atrio a Mosaico (IV, 1-2), ed in particolare della sua esedra 9 (fig. 5).

\section{A scala di insula: I'insula III, dai cunicoli borbonici all'archeologia virtuale}

La scelta dell'insula cui dedicare uno studio di dettaglio per il progetto DHER è caduta sull'insula III, in ragione sia della sua "seconda vita", e quindi della sua storia conservativa, dalle prime esplorazioni ad oggi, sia della sua storia in antico, che l'assetto attuale (tredici unità edilizie, diverse nelle dimensioni, nell'articolazione degli spazi e nella qualità abitativa) lascia immaginare complessa e molto articolata ${ }^{19}$.

Già intercettata dalle indagini borboniche, l'insula appartiene al primo settore dell'antica Ercolano interessato dagli scavi a cielo aperto, sotto la direzione di C. Bonucci, negli anni 1830-1831.

A questo primo esperimento, limitato all'esplorazione parziale della Casa dello Scheletro (III, 3), fece seguito una lunga cesura negli interventi sul terreno, fino alla ripresa, da parte di A. Maiuri e ad iniziare dal 1927, degli scavi, che fra 1928 e 1929 portarono al completo recupero, e al restauro, dell'intera insula.

Nell'assetto museografico voluto da A. Maiuri l'insula si articola in almeno tredici unità edilizie, di diversa natura e vocazione, dalla grande e lussuosa villa urbana nota come Casa dell'Albergo (III, 1. 19) alle abitazioni di minor estensione ma comunque riccamente decorate, con scelte dietro alle quali si possono forse intravedere le esigenze di un ceto medio desideroso di emulare il modus vivendi delle classi più elevate, quali la Casa dello Scheletro (III, 3) e la Casa dell'Erma di Bronzo (III, 16), fino alla Casa a Graticcio (III, 13. 14), risposta a bisogni abitativi ancora diversi, di utenti dalle minori possibilità di spesa.

Non solo, quindi, l'insula è stata interessata da indagini più o meno estese in tutte le fasi del recupero del sito di Ercolano, dai cunicoli borbonici fino ai primi scavi a cielo aperto e poi agli scavi estensivi di A. Maiuri, ma offre anche un'evidenza materiale molto articolata: le unità che la costituiscono, molto diverse l'una dall'altra, per quanto riguarda sia le strutture che le decorazioni, presentano una complessa storia edilizia e conservativa, caratterizzata da una fitta rete di ristrutturazioni, restauri e cambiamenti di destinazione d'uso, ed una fisionomia decorativa altrettanto diversificata.

19 Sull'insula III, A. MaIURI, Ercolano. I nuovi scavi (1927-1958), Roma, 1958 ; De Kind, 1998, pp. 90-130; Самаrdo,Nотомista, 2017, pp. 183-194. 
Appartengono infatti a quest'insula due dei tre casi di mosaici parietali noti ad Ercolano, mentre l'insieme delle sue pareti dipinte offre un articolato campionario della tradizione pittorica dei siti vesuviani, dal II stile del settore termale della Casa dell'Albergo (III, 1, 19) al III stile della Casa dell'Erma di Bronzo (III, 16) e allo stile transizionale, fra III e IV, della Casa del Tramezzo di Legno (III, 4, 11).

Questo insieme di spazi costruiti, decorati e fruiti, risultato di un complesso intreccio di azioni susseguitesi in un lungo arco cronologico, fra antico e moderno, è stato indagato, nell'ambito del progetto DHER, in tutti i suoi aspetti, attraverso un modus operandi affinato negli anni.

Alle prime esperienze di rilievo planoaltimetrico (2005-2006) e fotogrammetrico (2005-2013) hanno fatto seguito, nel 2016, nuovi interventi aggiornati nel metodo e nelle tecniche, che hanno consentito di perfezionare i risultati già ottenuti e di porre le basi per ulteriori progressi.

Il rilievo planoaltimetrico realizzato con Total Station, sul quale si erano fondate anche le sperimentazioni di archeologia virtuale, realizzate fra 2008 e 2010 in collaborazione con il CINECA sul caso di studio della Casa dello Scheletro (III, 3) ${ }^{20}$, è stato infatti nel 2016 rivisto ed aggiornato, a cura del CISA dell'Università di Napoli L'Orientale, sulla base di una nuova e completa campagna di acquisizione dei dati per il tramite di un rilievo tridimensionale, realizzata da un'équipe mista dei due Atenei (fig. 6) ${ }^{21}$. In maniera complementare, nel rilievo fotogrammetrico, finalizzato alla realizzazione di un corpus di immagini metricamente corrette sulle quali fondare l'analisi e l'interpretazione degli elevati e dei rivestimenti, dal prodotto della prima stagione (fotopiani) si è passati ad un altro (ortofoto), che offre un risultato di maggiore qualità.

Per quanto concerne gli apparati decorativi, il lavoro dell'Atlante può oggi dirsi completo (o quasi, con la sola eccezione dell'inaccessibile Casa a Graticcio III, 12-13) per l'insula in esame, sia nella documentazione fotogrammetrica, sia nella restituzione grafica e nel catalogo, con particolare riguardo per gli ornati parietali: decine di superfici-limite verticali, a cui si aggiunge un numero più ridotto di rivestimenti pavimentali, il tutto organizzato in un apposito database.

Su questo peculio di dati, integrato dai risultati delle ricerche di archeometria della pittura parietale (confocus sui pigmenti, e quindi sulla tavolozza cromatica), si è fondato lo studio dell'insieme decorativo nel suo contesto, dal livello urbanistico a quello architettonico e funzionale.

20 A. Coralini et alii, 2010.

21 Coerentemente con la tradizione e la vocazione del programma Vesuviana e dei suoi progetti, ai ricercatori "anziani" si sono affancati, in tutte le fasi della linea di azione, dall'acquisizione dei dati alla post-produzione, giovani archeologi in formazione, fra cui si è segnalato, per l'importanza del suo contributo, soprattutto Gabriele Boi. 


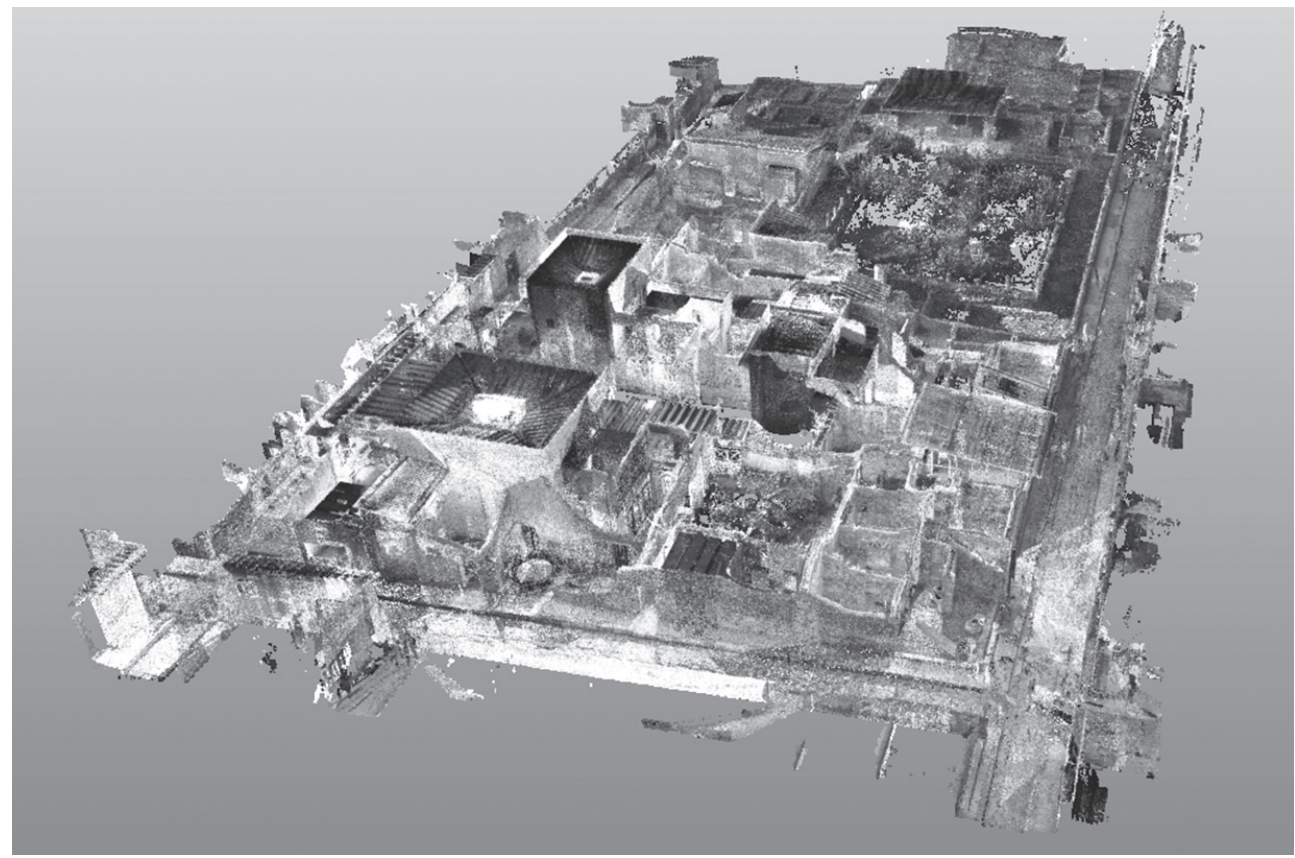

Fig. 6. Ercolano, insula III. Modello tridimensionale (Programma Vesuviana, DHER; Università di Napoli L'Orientale, CISA, 2016).

Per la lettura dell'organismo edilizio dell'insula e delle sue componenti in antico, e per la migliore comprensione delle sue vicende post-scavo, accanto ai dati delle campagne di rilievo integrato, un fondamentale contributo è venuto dall'analisi comparativa della documentazione d'archivio, testuale ed iconografica: da una parte, i Giornali degli Scavi (1927-1961) e i Giornali dei Lavori (1929-1987), fonte preziosa di informazioni sullo stato di avanzamento dei lavori di scavo e sugli interventi di consolidamento e restauro spesso concomitanti con le operazioni di recupero, che possono consentire di meglio leggere e interpretare l'evidenza materiale attuale, frutto dell'immagine di città che A. Maiuri volle restituire; dall'altra, i materiali conservati nell'Archivio Fotografico e dei Disegni, che di quelle informazioni costituiscono spesso un'utile integrazione ${ }^{22}$.

La storia conservativa dell'insula III, scelta come caso di studio del progetto DHER anche per il fatto di essere stata interessata da tutte le principali fasi di scavo dell'antica Ercolano, inizia negli anni delle esplorazioni in cavo cieco, durante la stagione borbonica, quando l'insula viene intercettata dalla rete di

22 Recente è il riconoscimento delle potenzialità informative di una fonte d'archivio generalmente trascurata, Sorbo, 2014; Camardo, Nomista 2017. 
cunicoli e spogliata degli oggetti e degli apparati decorativi di maggio pregio, come ben dimostra l'esempio della Casa dello Scheletro (III,3) e del suo ninfeo grande, asportato e trasferito nelle collezioni reali ${ }^{23}$. Dal 1830 l'insula viene parzialmente riportata in luce da C. Bonucci, con interventi di scavo a cielo aperto che a causa delle difficoltà tecniche e dei costi hanno vita breve, senza essere seguiti da consistenti interventi conservativi: una situazione che ha determinato la perdita fisica delle decorazioni parietali negli ambienti esplorati in quell'occasione, perdita aggravata dall'assenza di documentazione di dettaglio, sia grafica che testuale ${ }^{24}$. Dopo altre indagini non sistematiche nel corso dell'Ottocento, il completamento della sua esplorazione, così come il suo restauro, si deve all'intervento di A. Maiuri, fra il 1927 e il 1929.

Nell'insula sono tuttora visibili i segni delle vicende, anche moderne, che hanno determinato il suo aspetto attuale. Esemplare in tal senso è la Casa dello Scheletro (III, 3), dove sono ancor oggi riconoscibili alcuni interventi anteriori ai Nuovi Scavi di A. Maiuri, attribuibili, piuttosto che ai primi anni dell'avvio degli scavi a cielo aperto (1828-1931), sotto la direzione di C. Bonucci, o agli anni immediatamenti successivi il 1850, quando C. Bonucci rientrò ad Ercolano come Architetto Locale, a quelli della Direzione di G. Fiorelli (1869-1877). Questi lavori di "riparazione", che procedono di pari passo con quelli di "cavamento", consistevano soprattutto in operazioni di distacco e conservazione degli intonaci, di protezione delle pareti affrescate, di ripristino delle aperture e di consolidamento ${ }^{25}$. Nella Casa dello Scheletro, in particolare, le pitture dell'atrio e del settore a ovest di questo, riportati alla luce fra 1830 e 1831 dagli scavi di C. Bonucci, sono andate completamente perdute, mentre restano leggibili le pitture dei settori centrale e orientale, recuperati un secolo dopo dagli scavi di A. Maiuri, che provvide al consolidamento delle strutture antiche e alla ricomposizione degli apparati decorativi parietali, danneggiati dall'eruzione e dall'attività di scavo dei secoli

23 M. Pagano, Metodologia dei restauri borbonici a Pompei ed Ercolano, in Rivista di Studi Pompeiani, 5 (1991), p. 167-191; ID., Calco, ricomposizione e restauro del ninfeo della Casa dello Scheletro di Ercolano, in F. Guidobaldi, A. Paribeni (a cura di), Atti del V Colloquio dell'Associazione Italiana per lo Studio e la Conservazione del Mosaico (Roma, 3-6 novembre 1997), Ravenna, 1998, p. 337-344; M.S. Pisapia, Particolari inediti del ninfeo grande della Casa dello Scheletro di Ercolano, in C. Angeleldi, A. PAribeni (a cura di), Atti del XII Colloquio dell'Associazione Italiana per lo Studio e la Conservazione del Mosaico (Padova-Brescia, 14-17 febbraio, 2006), Ravenna, 2007, p. 597-605.

24 Sulla possibilità di individuare, attraverso l'esame comparato dello stato dei luoghi attuale e delle fonti d'archivio, gli interventi conservativi anteriori ai restauri di A. Maiuri, op.cit. Sorbo, 2014, pp. 187-197.

25 Sorbo, 2014, p. 192. 
precedenti, procedendo anche al ripristino delle coperture, oltre che ad alcuni interventi di musealizzazione in situ ${ }^{26}$.

Sulla linea delle esperienze compiute nel progetto pompeiano (1999-2002), anche nell'ambito del progetto DHER la collaborazione con il Vis.I.T. Lab del CINECA è stata finalizzata alla sperimentazione di un modello tridimensionale, filologico ed interattivo, utilizzabile come interfaccia per l'archiviazione e la gestione dei dati di un progetto di ricerca. La sperimentazione, sviluppata fra 2008 e 2010, ha condotto alla realizzazione di un prototipo sul caso di studio della Casa dello Scheletro (III, 3), per il tramite di un software Open Source, VisMan ${ }^{27}$.

\subsection{Documentare l'esistente: spazi costruiti e spazi decorati}

Coerentemente con la vocazione alla sperimentazione aperta e continua propria del programma Vesuviana, l'ìnsula III di Ercolano è diventata per il progetto DHER un cantiere-laboratorio nel quale mettere a punto metodi e tecniche di intervento per una sempre più approfondita e aggiornata conoscenza del caso di studio, con un particolare interesse per il rapporto fra spazi e decorazioni e per il modo in cui queste ultime modulano le architetture e la loro fruizione.

\subsubsection{Le strutture}

$\mathrm{Al}$ rilievo con stazione totale e al rilievo fotogrammetrico finalizzato alla realizzazione di fotopiani, in uso fra 2005 e 2015, nel 2016 si è sostituito un nuovo e integrale rilievo, tridimensionale (con il Laser Scanner Faro Focus 3D X130), dell'insula III, realizzato, sotto la direzione di Andrea D'Andrea, da un'équipe mista, dell'Università di Napoli L'Orientale, con il suo CISA, e dell'Università di Bologna, con il suo Laboratorio di Archeologia dell'Architettura ${ }^{28}$ (fig. 6). Il risultato consiste in 329 scansioni, di cui 23 per l'inquadramento topografico dell'area e 306 per gli ambienti delle singole unità edilizie, per una superficie di poco superiore a 4.000 mq, articolata in 13 unità e 243 ambienti.

Per migliorare la fase di registrazione automatica delle riprese sono stati attivati i sensori relativi alla bussola, al piano inclinato ed all'altimetro. Il dato RGB è stato acquisito considerando generalmente una luce proveniente dai lati di ripresa, piuttosto che dall'alto.

26 Camardo, Notomista, 2017.

27 Coralini et alii, 2010.

28 Per una prima presentazione dei risultati di questa attività, A. D'Andrea et alii, Vesuviana, DHER. Ercolano, insula III: rete topografica 3D per lo studio e la tutela programmata, poster presentato al convegno “Ricerca e valorizzazione» (Bologna, 4-6 maggio 2017). 
Per georiferire le nuvole di punti è stata realizzata una prima serie di riprese finalizzata a circoscrivere l'area dell'insula. Sono state quinfi eseguite 23 scansioni lungo la viabilità esterna con punti di ripresa ogni 10 metri circa ed in corrispondenza dell'ingresso delle differenti UUFF in modo da semplificare la successiva fase di allineamento delle nuvole relative alle singole Domus.

Con la stazione totale sono stati misurati 10 target, partendo da una serie di vertici di primo livello della rete topografica disposti lungo la viabilità, sulla base delle monografie dei punti messe a disposizione dall'Herculaneum Conservation Project. I Ground Control Points (GCPs), espressi in coordinate geografiche Gauss-Boaga Fuso Est, sono stati ripresi soltanto da due distinte scansioni.

Successivamente sono state realizzate 306 scansioni per gli ambienti delle 13 unità funzionali (UF). Le attività di ripresa sono state organizzate per UF e, per garantire il corretto allineamento di tutti i modelli, le scansioni di ciascuna UF sono state realizzate partendo dalla viabilità esterna. Particolare cura è stata dedicata alla acquisizione degli elementi di raccordo dell'UF 10 (Casa dell'Albergo), in relazione ai passaggi di accesso ai vari livelli di frequentazione dell'edificio.

$\mathrm{Al}$ termine delle riprese si è proceduto con il processamento e l'allineamento delle scansioni con il software Scene, ad iniziare dalla rete di inquadramento, le cui scansioni sono state allineate in base agli algoritmi "vista dall'alto" e da "nuvola a nuvola", che hanno consentito di avere un errore di registrazione medio di mm 2 (77\% dei punti al di sotto di $4 \mathrm{~mm}$ di errore). Grazie ai GCPs la rete è stata poi roto-traslata secondo coordinate polari.

In una seconda fase, ciascuna unità funzionale è stata processata singolarmente e poi registrata sulla rete 3D. Per ottimizzare l'accesso ai singoli edifici, all'interno del software Scene, sono state create singole cartelle relative alle rispettive unità funzionali. Ciascuna cartella è poi stata ottimizzata eliminando i punti duplicati senza eccessiva decimazione dei modelli. In questo modo si è resa possibile la navigazione nell'intero modello dell'insula, nei singoli edifici o nei singoli ambienti, estraendo per ciascun punto le relative coordinate assolute nel sistema Gauss-Boaga, oltre che l'estrapolazione dei foto-ortopiani di pareti affrescate o delle tessiture murarie.

L'intero modello georiferito è stato poi esportato in ReCap e sovrapposto, in ambiente CAD, alla planimetria di dettaglio eseguita dall'équipe dell'Herculaneum Conservation Project, in modo da verificare la corretta rototraslazione del rilievo tridimensionale.

Per completare il rilievo, con l'acquisizione dei tetti e delle creste murarie, è stata effettuata una campagna complementare di riprese, aerofotogrammetriche, con il drone Matrice 100, da un'altitudine di 15 m e garantendo una sovrapposizione di circa l'85 per cento tra i singoli scatti. Per la restituzione del settore meridionale della Casa dell'Albergo(III, 1, 10) (un'area di 50 x 49 m) sono state necessarie oltre 140 immagini e dieci minuti di volo, con una risoluzione di circa $4 \mathrm{~mm}$ Pixel, tale 
da assicurare una corretta lettura anche delle pavimentazioni degli ambienti scoperti (fig. 6).

Il rilievo tridimensionale così ottenuto offre la possibilità di georiferire e scalare correttamente le ortofoto ottenute con tecnica di fotogrammetria SfM per gli ambienti decorati degli edifici. Al fine di permettere l'analisi di dettaglio degli ornati, per i foto ortopiani si è scelta una distanza di ripresa in media di $2.5 \mathrm{~m}$ dall'oggetto, tale da consentire di generare un modello ad altissima risoluzione fotografica.

La rete topografica tridimensionale potrà inoltre essere utilizzata anche come griglia di riferimento per ulteriori e future acquisizioni fotogrammetriche, consentendo di monitorare periodicamente lo stato di conservazione delle tessiture murarie, degli elementi architettonici e delle superfici decorate.

\subsubsection{Gli apparati decorativi.}

Nell'ambito delle due principali linee di azione del progetto DHER, l'Atlante degli Apparati Decorativi e lo studio di dettaglio dell'insula III, la campagna 2016 è stata dedicata, anche in questo caso grazie alla collaborazione fra il Laboratorio di Archeologia dell'Architettura dell'Università di Bologna e l'équipe del CISA (Centro Interdipartimentale di Servizi per l'Archeologia) dell'Università di Napoli L'Orientale, all'integrale revisione e alla nuova acquisizione dei rilievi fotogrammetrici delle superfici verticali (pareti) e orizzontali (pavimenti) dell'insula III (fig. 1-2), cui ha fatto seguito, nei mesi successivi, l'indispensabile lavoro di post-produzione ${ }^{29}$.

Lo scopo finale dell'intervento, in piena coerenza con la linea di azione dell'Atlante degli Apparati Decorativi, era quello di fornire agli "specialisti di settore", ovvero agli archeologi e disegnatori specializzati nell'analisi e nella restituzione degli apparati decorativi, una base documentaria metricamente corretta, fotogrammetrica, per il rilievo vettoriale e la ricostruzione grafica delle decorazioni parietali. Di conseguenza, essendo la necessità primaria quella di poter distinguere il massimo numero possibile di caratteri della superficie pittorica, la ricchezza di dettagli doveva essere il primo requisito delle immagini da produrre. La pianificazione del rilievo fotogrammetrico si è quindi fondata sulla necessità di rispondere a tale esigenza.

In prima istanza, si sono eseguiti alcuni test, per la definizione della distanza di presa ottimale.

29 Per una prima presentazione dei risultati di questa attività, A. Fiorini, Vesuviana, DHER. Ercolano, insula III: il rilievo fotogrammetrico delle superfici decorate, poster presentato al convegno «Ricerca e valorizzazione» (Bologna, 4-6 maggio 2017). 
In seguito, nelle riprese fotografiche e nei relativi prodotti fotogrammetrici, si sono valutati il grado di nitidezza e la dimensione dei dettagli d'interesse (motivi decorativi ed elementi figurati). Si è potuto così stabilire che la fotocamera in dotazione (Nikon D7100, munita di obiettivo Nikkor AF-S 18-70 millimetri) era in grado di fornire immagini utili al lavoro di archeologi e disegnatori se la distanza di presa non superava i 2,5 metri per le pareti e i 5 metri per i pavimenti. A queste condizioni le "immagini-test" apparivano infatti molto ricche di dettagli, dato che ogni pixel "catturava" una porzione quadrata di parete grande solo 0,5 millimetri di lato (1 mm per i pavimenti).

Una volta definito il metodo di lavoro, le superfici da documentare sono state annotate sulla planimetria e associate a un codice identificativo.

Completata questa prima fase, si è eseguito un sopralluogo finalizzato alla valutazione delle condizioni di accessibilità, illuminazione, estensione e conservazione di ogni superficie. In questo modo è stato possibile stabilire per ognuna di esse la migliore situazione di ripresa (l'ora della giornata più adeguata alle riprese fotogrammetriche; la necessità o meno di eseguire operazioni di ripulitura da polvere e/o erbe infestanti; la necessità o meno di impiegare fonti di luce ausiliarie; la necessità o meno di posizionare la fotocamera sull'asta telescopica o sul semplice treppiede).

Presentando gli ambienti una illuminazione naturale troppo debole, oppure poco uniforme, si è reso necessario il ricorso a fonti di luce artificiale, senza le quali molte superfici non si sarebbero potute rilevare. Per ovviare alle difficoltà di messa a fuoco della superficie da parte della fotocamera in ambienti in condizioni di illuminazione non naturale, fatica a trovare la corretta messa a fuoco della superficie, si è fatto ricorso a lampade a luce continua, disposte in maniera tale da ottenere una illuminazione diffusa e omogenea su tutta la superficie. Inoltre, quando possibile, si sono oscurate le fonti di luce naturale degli ambienti, quali porte e finestre, per impedire che la luce naturale (quasi sempre di tonalità differente rispetto a quella artificiale) introducesse variazioni cromatiche della superficie pittorica, vanificando la corrispondenza ricercata, in primo luogo, con il bilanciamento del bianco e successivamente con il target di colori codificati.

In questo specifico settore di studi la fedeltà cromatica delle immagini è infatti un parametro di fondamentale importanza, che deve sempre essere tenuto in considerazione. Per questo motivo, dopo aver ottimizzato l'illuminazione della parete da documentare, si sono eseguite alcune riprese ponendo in campo il target di grigio neutro e successivamente il ColorChecker Passport. Compiuta questa operazione, è stato possibile procedere al rilievo fotogrammetrico, avendo cura di verificare sempre che le condizioni di luce non subissero variazioni.

Per garantire stabilità in ripresa, ridurre il fenomeno del micromosso e ottenere la massima nitidezza di dettaglio possibile, è stato utilizzato un treppiede. In alternativa, per le porzioni di parete non raggiungibili dall'operatore (e per i 
pavimenti), la fotocamera è stata fissata sulla cima di un'asta telescopica con estensione massima di 8 metri.

Nel rilievo fotogrammetrico il controllo dell'inquadratura è un'operazione tecnica che deve essere condotta con molta attenzione, in quanto può incidere sulla qualità metrica degli elaborati finali, in quanto per rilevare una parete servono, in primo luogo, inquadrature a piano verticale e il più possibile frontali. Nell'insula III di Ercolano è stato impiegato un sistema di controllo già collaudato: un piccolo dispositivo connesso alla fotocamera (Nikon WU-1a) consente a qualsiasi smartphone di visualizzare la scena inquadrata e di scattare la foto.

A conclusione del rilievo, per ogni superficie documentata, si sono eseguite le consuete fotografie d'archivio, una sorta di giornale di bordo in immagini, in cui devono comparire la palina e la lavagna (dove vengono indicati il giorno di esecuzione del rilievo e il codice identificativo del pavimento o della parete).

Per consentire agli esperti di restituzione grafica delle decorazioni antiche di ottenere dalle immagini informazioni corrette circa la forma e le dimensioni dei motivi decorativi e degli elementi figurati, è stato infine necessario ricorrere ad una rielaborazione informatica in grado di trasformare le normali fotografie in ortofoto (nel caso specifico, tramite il software Agisoft PhotoScan Professional).

In totale si sono eseguiti ex novo i rilievi e le ortofoto di 33 superfici (24 pareti e 9 pavimenti), che, aggiungendosi alla revisione del materiale già acquisito in precedenza (2005-2015), hanno consentito di completare, per la parte fotogrammetrica, la sezione dell'Atlante degli Apparati Decorativi di Ercolano corrispondente all'insula III.

Sulla base di questo corpus di immagini metricamente corrette, e con il supporto del riesame dei documenti fotografici d'archivio e delle verifiche autoptiche di volta il volta necessarie, è stato possibile, inoltre, procedere alla completa revisione delle restituzioni grafiche delle decorazioni parietali oggi ancora in situ, ove possibile integrate con quelle dei disiecta membra individuabili nelle raccolte del Museo Archeologico Nazionale di Napoli ${ }^{30}$.

30 I risultati di questo approccio integrato allo studio e alla valorizzazione degli apparati decorativi dell'insula III saranno presentati nel secondo volume del progetto DHER, di prossima pubblicazione (Spazi e decorazioni. L'insula III). Per un primo tentativo di restituzione e catalogazione delle pitture parietali della medesima insula, oltre ai lavori degli allievi del Laboratorio di Rilievo e Restituzione della Pittura Antica, che ne hanno interessato quasi tutte le unità edilizie, con l'eccezione della Casa a Graticcio: L. Galvagni, "Il settore atriense della Casa del Tramezzo di Legno ad Ercolano. Rilievo e ricostruzione della facciata» (tesi di laurea magistrale, Università di Bologna, Facoltà di Lettere e Filosofia, a.a. 2011-2012; relatore, Daniela Scagliarini) e C. Condoni, «Pitture e mosaici di Ercolano. Rilievo, analisi, ricostruzione, contestualizzazione» (tesi di diploma, Dottorato di Ricerca in Scienze Archeologiche, Università di Padova, XXVIII ciclo, 2013-2015; tutor, Antonella Coralini). 
Per il caso specifico dell'insula III, e per i suoi apparati decorativi strutturali (parietali e pavimentali), l'analisi comparata delle fonti testuali e di quelle iconografiche ha consentito di restituire ai contesti di rinvenimento, e quindi di destinazione e fruizione in antico, almeno sette disiecta membra oggi conservati nelle raccolte del Museo Archeologico Nazionale di Napoli, fra cui anche la zona superiore della decorazione della parete nord dell'ala della Casa del Tramezzo di Legno $(\mathrm{III}, 4,12)^{31}$.

\subsection{Archeologia degli archivi e dei depositi, fra contesti moderni, di rinvenimento, e contesti antichi, di destinazione e fruizione}

Come ben dimostrato dalle ricerche condotte nell'ultimo mezzo secolo, con focus sulla pittura parietale, il potenziale informativo delle indagini integrate in archivio e in deposito è per il sito di Ercolano particolarmente interessante ${ }^{32}$.

Se l'individuazione del contesto di appartenenza può considerarsi altamente affidabile per gli elementi staccati e trasferiti altrove degli ornati fissi, ovvero dei rivestimenti delle pareti e dei pavimenti, sulla base sia dei dati offerti dalle fonti documentarie, sia dell'esame comparato con quanto ancora si conserva in situ, alla ricerca delle affinità formali che possano fungere da indicatori per quella reductio ad unum, il margine di sicurezza delle ipotesi di lavoro si riduce di molto, nel caso di Ercolano, quando l'oggetto di interesse è costituito dai reperti mobili, e quindi da arredi, suppellettili e oggetti riconducibili alla cultura materiale, componenti fondamentali della scena domestica antica ${ }^{33}$.

Anche per i documenti di maggior pregio, oggetto in genere di maggior attenzione sia nella fase di rinvenimento sia nella conservazione, quale la scultura decorativa, nella forma sia di statue e rilievi sia di arredi configurati, il livello di affidabilità delle ipotesi di restituzione ad uno spazio specifico, ad un edificio

31 MANN, inv. 10008 (la nicchia decorata a mosaico dalla Casa dello Scheletro, III, 3); MANN, inv. 8592 (parte della zona superiore dell'ala 5 della Casa del Tramezzo di Legno, III, 4-12); MANN, inv. 9727; MANN, inv. 9741; MANN, inv. 8609; MANN, inv. 9730; MANN, inv. 9419 (dalle fauces della Casa dell'Erma di Bronzo III, 16), come già indicato in M. HinterhöLler, «Die Wandmalereien des Dritten Stils in der Casa dell'Erma di Bronzo in Herkulaneum und ein Landschaftsfresko im Nationalmuseum von Neapel», in RHistM 50 (2008), p. 69-160. Per le pitture alibi riconducibili all'insula III, A. Allroggen-Bedel, nel volume di prossima pubblicazione del progetto DHER (Spazi e decorazioni: l'insula III).

32 A. Allroggen-Bedel, “Ercolano. Dal sito agli archivi, dal museo al sito», in Vesuviana, 2009, p. 167-179, con bibliografia precedente; D’Alconzo, 2002.

33 Per la cultura materiale dall'insula III, gli studi sono ancora in corso: per un primo approccio, C. Ascari Raccagni, Ceramica a pareti sottili da Ercolano in DHER, 2011, pp.371-396. 
preciso ed un ambiente, è per Ercolano fortemente limitato da due gravi ostacoli: da una parte, dalla laconicità e genericità delle relazioni di scavo ${ }^{34}$; dall'altra, dalle modalità del seppellimento del sito di Ercolano, così violento e traumatico da rendere poco attendibili le stesse indicazioni di rinvenimento ${ }^{35}$.

Nel corso dello studio di dettaglio dei resti di apparati decorativi scultorei rinvenuti nell'insula III di Ercolano ${ }^{36}$, è emersa con evidenza la difficoltà di reperire, nella documentazione anteriore al 1927, anno di avvio della lunga stagione di scavi a cielo aperto diretta da A. Maiuri, dati che colleghino in modo certo reperti non più in situ a contesti di quest'insula. Diversa è invece la situazione per le indagini del Novecento, non ricchissime ma comunque meno avare di informazioni. In questo senso risultano fondamentali i Giornali degli Scavi (1927-1961), conservati in duplice copia (ad Ercolano e presso l'Archivio del Museo Archeologico Nazionale di Napoli), che forniscono dati circa il rinvenimento dei singoli oggetti, dati preziosi ma comunque da valutare con le cautele imposte dalla forza con cui il flusso piroclastico dell'eruzione del 79 d.C. dovette trascinare anche per più metri non solo gli oggetti, tanto più se di medie e piccole dimensioni, ma anche, in certi settori, le strutture.

Nell'insula III, il grande complesso che ne occupa l'intero settore meridionale e che parte della letteratura ritiene una villa urbana, al pari della Casa dei Cervi (o della Casa del Rilievo di Telefo), la Casa dell'Albergo (III, 1. 19), spogliata negli scavi settecenteschi e ottocenteschi, non ha restituito arredi figurati durante gli

34 Sulle potenzialità informative degli archivi di scavo, non solo per gli anni della Direzione di Amedeo Maiuri, ma anche per le stagioni precedenti, dagli interventi borbonici in poi (per le quali resta di fondamentale importanza la raccolta critica curata da M. Ruggiero, Storia degli scavi di Ercolano ricomposta sui documenti superstiti, Napoli, 1885, cui si sono aggiunti ulteriori contributi, quali quelli di U. Pannuti, Il Giornale degli Scavi di Ercolano (1738-1756), in Memorie dell'Accademia dei Lincei, 26 (1983), p. 163-410; M. Pagano, I diari di scavo di Pompei, Ercolano e Stabiae di Francesco e Pietro La Vega (1764-1810). Raccolta e studio di documenti inediti, Roma, 1997; ID., I primi anni degli scavi di Ercolano, Pompei e Stabia. Raccolta e studio di documenti e disegni inediti, Roma, 2005); da ultimo, N. MonteIx, "Exploiting excavation archives: Herculaneum from 1927 to 1961 as a case-study", in Journal of Roman Archaeology 29 (2016), p. 423-437, che riprende e sviluppa le riflessioni già presentate in ID., Inventio Herculaneis. Per la rilettura dei giornali di scavo di Ercolano, in Vesuviana, 2009, p. 181-196, e ID., Les lieux de métier: boutiques et ateliers d'Herculanum, BEFAR 344, Rome, 2010. Sull tema Maiuri, 1958, pp. 23-26. Lo studio, di prossima pubblicazione nel secondo volume dedicato al progetto DHER (Spazi e decorazioni: l'insula III), è stato condotto da Luca Di Franco (sculture) e Rocio Manuela Quadra Rubio (arredi). 
scavi del Novecento, a differenza di quanto avvenuto per gli interventi precedenti. Dall'esame dei diari di scavo di C. Bonucci si apprende, infatti, che nel 1852 il complesso fu interessato dal ritrovamento di cteste di due statue di marmo, l'una di un Fauno coronato di pino, l'altra di un giovane Mercurio con capelli ricci e piccole alette sulla fronte": subito donate agli ospiti del re in visita al cantiere, i "Gran Duchi di Russia», le due teste sono oggi irreperibili, ma la pur laconica descrizione ottocentesca fa comunque ritenere che si trattasse di sculture da giardino.

Un'analoga penuria di reperti caratterizza anche l'abitazione seconda per estensione nell'insula, cioè la Casa del Tramezzo di Legno (III, 4. 11), che si segnala solo per il rinvenimento di una pregevole trapeza.

L'unità edilizia che nell'insula III ha restituito il maggior numero di reperti riferibili al suo apparato decorativo non strutturale, è la Casa dello Scheletro (III, 3), che, intercettata dai cunicoli borbonici nel 1740-41 e poi scavata, negli anni 1830-31, da C. Bonucci, e successivamente nel 1869 e nel 1876, venne portata completamente alla luce nel 1927, sotto la direzione di A. Maiuri. L'abitazione, che nella letteratura scientifica è ritenuta il risultato dell'aggregazione, probabilmente in epoca giulio-claudia, di tre originarie unità edilizie orientate in senso nord-sud, era caratterizzata da istanze decorative di alto livello. Nel suo assetto attuale, frutto della restituzione determinata dai restauri di Amedeo Maiuri, appare evidente, nonostante i limiti imposti dalle ridotte dimensioni della superficie disponibile, la tendenza ad enfatizzare l'importanza degli spazi di rappresentanza e ricevimento, quali alle spalle del tablino, il triclinio absidato affacciato su un piccolo cortile con pseudo-ninfeo, nel settore occidentale, il triclinio abbinato al grande ninfeo che ne costituiva la quinta scenografica. In via ipotetica, accanto alle decorazioni musive di questo ninfeo, già riconosciute da R. De Kind come pertinenti alla Casa dello Scheletro (III, 3), e ai rinvenimenti citati da M. Pagano e R. Prisciandaro ${ }^{37}$, pare possibile ricondurre all'apparato decorativo di quest'abitazione due oscilla, recuperati anch'essi negli scavi borbonici. Nei diari di scavo del 24 gennaio 1741, come nei giorni e nei mesi precedenti, si elencano infatti i rinvenimenti pertinenti ad un medesimo cunicolo, fra cui «dos medallas circulares de marmol, de treze onz. ${ }^{\mathrm{s}}$ y media de diametro, que representan, en relieve, por la una parte, un muchacho á cavallo en un delphin, y por la otra un dragón", che, anche per l'assenza di altri oscilla che presentino una decorazione simile (da un lato, un Erote con doppio flauto su delfino e, dall'altro, un mostro marino), pare possibile riconoscere fra

37 R.E.L.B. De Kind, “Casa dello Scheletro at Herculaneum: the large Nymphaeum», in Cronache Ercolanesi 21 (1991), pp. 133-147; M. Pagano, R. Prisciandaro, Studio sulle provenienze degli oggetti rinvenuti negli scavi borbonici del regno di Napoli, Castellammare di Stabia 2006. 
le incisioni in rame realizzate per la pubblicazione delle Antichità di Ercolano esposte, ma poi rimaste inedite ${ }^{38}$.

Il 18 giugno 1927, nella medesima Casa dello Scheletro (III, 3), venne alla luce, ancora in situ, il primo reperto scultoreo dei Nuovi Scavi, un rilievo quadrangolare in marmo, decorato su un solo lato, con una figura di amorino in volo ${ }^{39}$. Musealizzato in situ, il rilievo, forse un oscillum, venne rinvenuto ad un'altezza di 4,04 $\mathrm{m}$ dal piano di calpestio, ancora imperniato sulla parete di fondo del piccolo cortile ornato da un elegante pseudo-ninfeo ad edicola e da pitture di giardino sulle pareti.

Sul lato opposto dell'insula III, anche la Casa a Graticcio (III, 13. 14) ha restituito più elementi d'arredo, tra cui due monopodia in marmo, uno per piano, e un oscillum frammentario, di soggetto mitologico (un centauro su un lato e un giovane sacrificante sull'altro), proveniente dall'ultima stanza del piano inferiore: vista la natura di spazio non residenziale dell'ambiente, nel 79 d.C. occupato da due vasche con residui di calce, è verosimile che si tratti solo del luogo di rinvenimento e non di quello di destinazione e d'uso in antico ${ }^{40}$. Al piano superiore dell'edificio, dotato di un accesso indipendente, in un ambiente interpretabile come cubicolo, furono ritrovate in un armadio in legno nove statuette in bronzo, il 9 e il 17 novembre 1928, a cui ne va probabilmente aggiunta una decima, già recuperata sul pavimento il 4 ottobre, tra il letto e la parete est.

Nella Casa dell'Erma di Bronzo (III, 16), una delle unità edilizie dell'insula III in cui meglio si sono conservati gli ornati parietali, inquadrabili nel III stile iniziale, le attestazioni relative all'arredo scultoreo sono molto ridotte. Si segnala, tuttavia, fra queste il rinvenimento di un busto ritratto in bronzo, al piano superiore, in un

38 Pagano, Prisciandaro, 2006, figg. 117-118.

39 Giornale degli Scavi di Ercolano, 18 giugno 1927: “alla profondità di m. 0.96 dalla sommità del muro, a m. 4.04 dal pavimento della casa, ed a m. 0.40 al di sopra della seconda tegola è uscita una lastra di marmo bianco quadrato con il lato di $\mathrm{m}$. 0.30. Essa è infissa nel muro mediante 4 chiodi di ferro. Sopra detto pezzo vi è un bellissimo altorilievo e credo che rappresenti un Amorino o Mercurio fanciullo? Tiene nella mano destra un caduceo? o fulmini? e nella sinistra una borsa. Inventario n. 41. L'amorino o Mercurio? Tiene le ali che attaccano alla testa e d'intorno ad esso svolazza un leggiero mantello. Il bassorilievo è rimasto in sito"

40 Giornale degli Scavi di Ercolano, 20 ottobre 1927: “Alla parete est di quest'ultima vasca trovansi infissa nel muro un medaglione di marmo. Inventario n. 70. Mancante della metà con bassorilievo di cui al lato A rappresenta la parte inferiore di un centauro e dalla parte opposta un giovane in atto di sacrificare dinanzi ad un' Ara votiva sulla quale è collocata una maschera barbuta forse divinità fluviale. L'ara votiva ha forma rettangolare sul lato anteriore trovasi scolpita un festone di fiori che attacca ai due lati estremi della parte alta. Essa misura m. 0.58 di diametro lo spessore è di m. 0.05". 
ambiente dotato di un ricco pavimento in sectile, forse un oecus ${ }^{41}$.

Nell'insieme, quanto noto dell'arredo in marmo, figurato e non, delle abitazioni dell'insula III appare di modesta entità e di qualità non elevata, forse in quanto espressione di una committenza di livello medio.

\subsection{Archeologia del restauro, fra fonti d'archivio e lettura degli alzati}

I restauri condotti da A. Maiuri, dettati spesso dalla necessità di ricostruire integralmente la parte inferiore degli edifici, danneggiati prima dalla catastrofe del 79 d.C. e poi dalle esplorazioni settecentesche, hanno comportato forti manomissioni sia delle strutture, sia dei rivestimenti parietali, che risultano spesso difficilmente individuabili, e solo sulla base di un attento riesame, cui anche il lavoro per l'Atlante degli Apparati Decorativi può dare un importante contribuito.

Di questa possibilità offre un buon esempio il caso del cubicolo (3) della Casa dell'Erma di Bronzo (III, 3), dove grazie al rilievo fotogrammetrico e alla restituzione grafica su quello fondata è stato possibile riconoscere un montaggio poco accurato delle pitture. L'ambiente, infatti, presenta una decorazione che, ad un attento esame autoptico, pare non coerente con i principi di simmetria e di analogia cui di norma si ispirava la sintassi decorativa delle superfici nella pittura parietale romana: l'attenta analisi dei moduli compositivi e la restituzione integrativa di quanto si conserva degli ornati hanno permesso di concludere che il mancato allineamento delle pitture ricollocate in situ è l'esito di un restauro non felice (fig. 7).

Di fondamentale importanza per una corretta e filologica archeologia del costruito, l'archeologia del restauro ha il compito di riconoscere le tappe e gli esiti di quel lungo e continuo processo di ibridazione fra antico e moderno che ha prodotto l'assetto attuale dei siti vesubiani. Fra i suoi strumenti più preziosi, accanto al Giornale degli Scavi (1927-1961) e alla documentazione fotografica d'archivio, sono i Giornali dei Lavori (1929-1987), che attendono ancora di essere pienamente valorizzati ${ }^{42}$. Questo registro degli interventi di manutenzione

41 Giornale degli Scavi di Ercolano, 15 febbraio 1929: “nell' angolo nord-ovest del $2^{\circ}$ piano fu raccolto l' Erma di bronzo descritta il 15/2/1929. Il pavimento è di pezzi di marmo di grandezze e colori diversi, predomina il giallo antico. L'ambiente misura m. 2.80 x 3.20». Sull 'erma, anche A. Carrella, L.A. D'Acunto, N. Inserra, C. SEPE, Marmora pompeiana nel Museo Archeologico Nazionale di Napoli: gli arredi scultorei delle case pompeiane, Roma, 2008, p. 171.

42 I Giornali dei Lavori, conservati presso l'Ufficio Scavi di Ercolano, consistono in 45 volumi e quaderni, ordinati cronologicamente e per tipologia di intervento, dall'ottobre 1929 all'aprile 1987. 

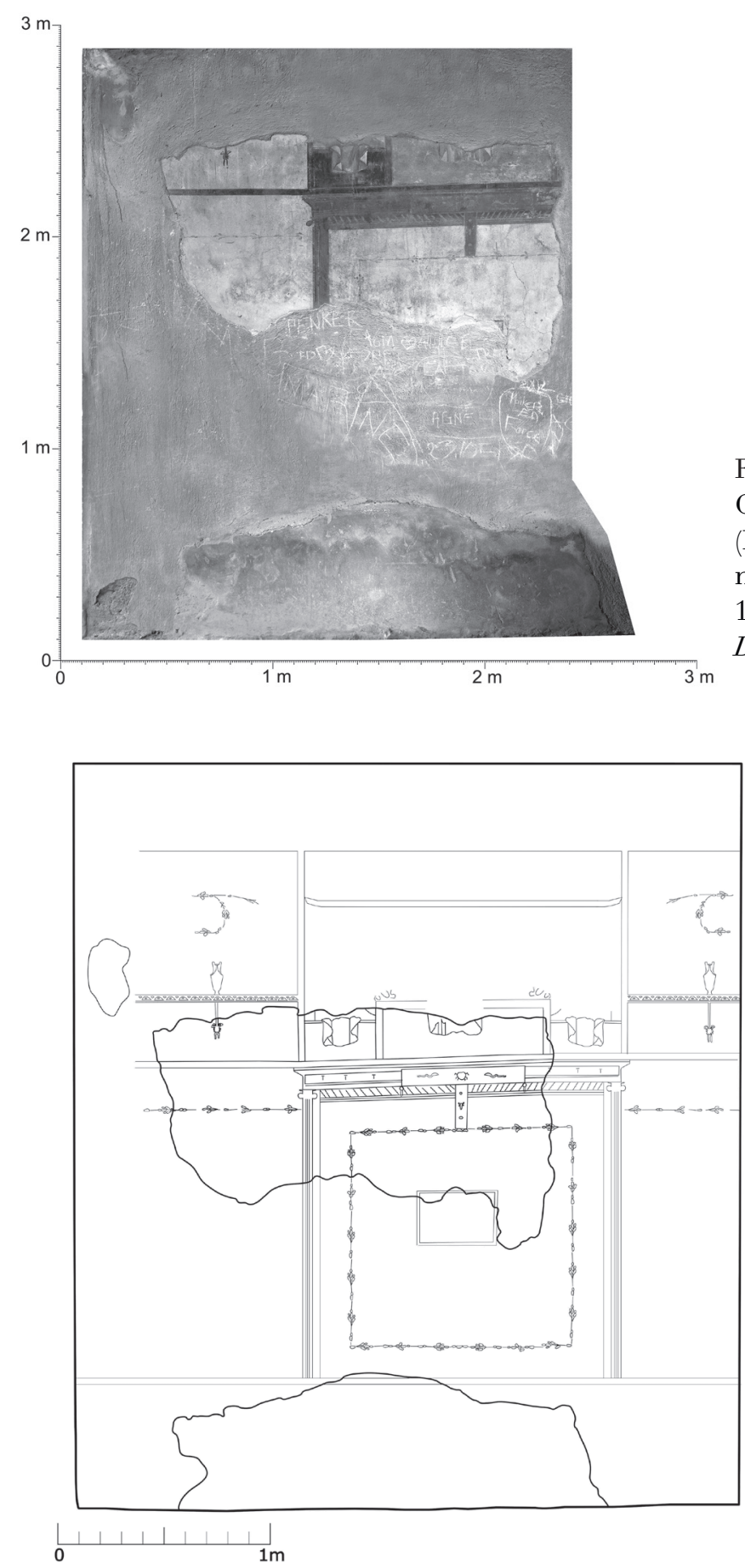

Fig. 7.a. Ercolano, Casa dell'Erma di Bronzo (III 16), cubicolo 3, parete nord: rilievo fotogrammetrico 1:20 (Programma Vesuviana, DHER, M. Zanfini, 2006).
Fig. 7.b. Ercolano, Casa dell'Erma di Bronzo (III 16), cubicolo 3, parete nord: rilievo integrativo lineare (Programma Vesuviana, DHER, L. Galvagni, C. Cordoni, 2011-2014). 


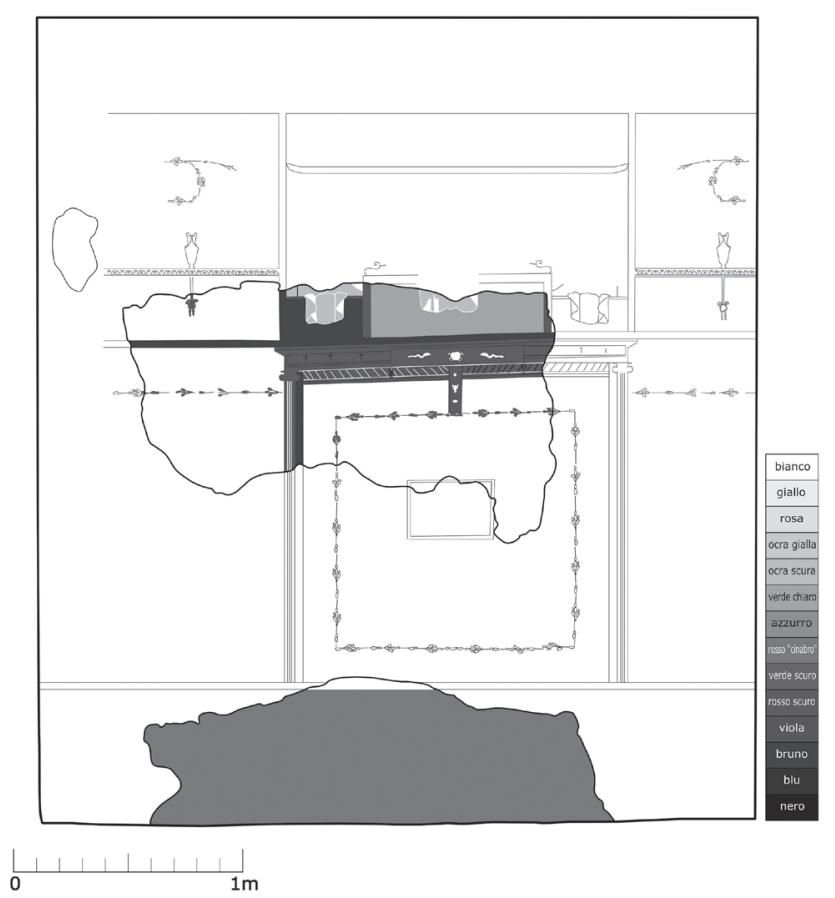

Fig. 7.c. Ercolano, Casa dell'Erma di Bronzo (III 16), cubicolo 3, parete nord: rilievo integrativo in scala di grigi (Programma Vesuviana, DHER, L. Galvagni, C. Cordoni, 2011-2014).

ordinaria che con regolarità si eseguivano nel sito, squadra per squadra, secondo le diverse specializzazioni, costituisce, per la conoscenza dei restauri novecenteschi, una fonte del tutto complementare ai Giornali dei Scavi, dando continuità e profondità alle operazioni cui si fa cenno nelle relazioni delle attività di scavo.

A partire dal mese di ottobre 1929 prende avvio negli Scavi di Ercolano la consuetudine di annotare, con periodicità quasi quotidiana, le operazioni di manutenzione e consolidamento condotte da scavatori e muratori, marmisti e stuccatori, conservatori, verniciatori e fabbri, che procedono alla pulizia, al consolidamento, al restauro ed al consolidamento di stucchi, mosaici, legni, suppellettili e, ovviamente, murature, spesso in simultanea con lo scavo. Alla base di questa rete sistematica e capillare di interventi stanno i criteri definiti da A. Maiuri, dall'impiego degli stessi materiali utilizzati in antico all'adozione di tessiture diverse da quelle "originarie nelle "murature di rifacimento", senza tuttavia trascurare un'omogeneità almeno cromatica, fino all'attenzione per il reperto peculiare degli scavi di Ercolano, la "materia lignea" ${ }^{43}$. 


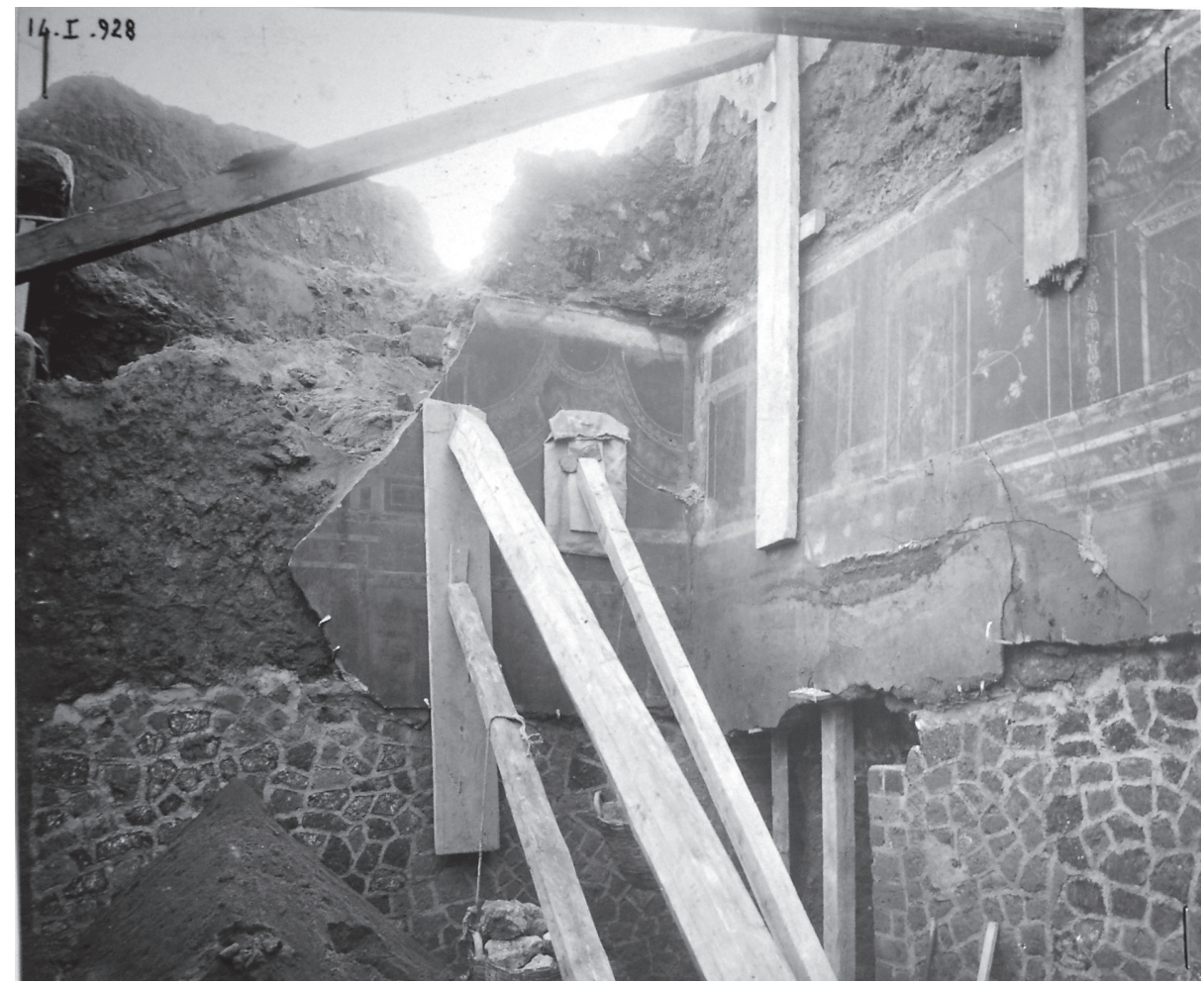

Fig. 8. Ercolano. Casa del Tramezzo di legno (III, 4. 11), triclinio (6). Scavi in corso e opere di restauro (1928): la parete tagliata nella zona inferiore dai cunicoli borbonici è assicurata alle murature di restauro (Archivio fotografico Parco archeologico di Pompei).

Nell'insula III alcune di queste opere di consolidamento e restauro appaiono ancora individuabili con un'osservazione attenta e consapevole.

Nella Casa dell'Albergo (III, 1. 19) e nella Casa annessa le murature marcite vengono demolite e ricostruite, secondo la presunta altezza originaria, ma vi si riconoscono anche riparazioni, qui eseguite in 'opera incerta' di tufo e cemento come legante e altrove, invece, come nella Casa dello Scheletro (III, 3), realizzate in 'opera listata' in tufo e laterizio e in 'opera reticolata'.

Diverso trattamento ricevono le strutture rinvenute durante gli scavi ottocenteschi, come quelle della Casa dell'Albergo (III, 1, 19), che vengono solo consolidate, con un getto di malta in lapillo o cemento.

Il problema delle pareti tagliate nella parte inferiore dai cunicoli borbonici, quali quelle del triclinio (6) della Casa del Tramezzo di legno (III, 4. 11), viene risolto in due tempi, prima con una tamponatura in opera incerta, assicurate con puntelli 
di legno, e poi procedendo al consolidamento e al collegamento a murature di restauro (fig. 8).

Per gli architravi di legno carbonizzato vengono adottate soluzioni diverse: ora sono sostituiti con elementi in ferro riempiti con muratura in opera incerta, come nelle Case dell'Albergo e dello Scheletro; ora si ricorre ad una formula più elaborata, che fa aderire alla parte di restauro parti originali in legno carbonizzato, consolidate con paraffina e olii minerali e protette da vetri.

Per i solai dei piani superiori, infine, si ricorre alla ricostruzione più o meno estesa, come ben esemplifica il portico (8) della Casa del Tramezzo di legno.

Alla protezione delle decorazioni parietali si provvede tramite coperture integrali - in ferro e vetro, nel cortile (27) della Casa dello Scheletro; in travi e tegole, nella Casa dell'Albergo; in cemento armato a finto legno, nella Casa del Tramezzo di legno - o, anche parziali, in altri casi, come nel triclinio (8) della Casa dell'Erma di bronzo, con tettoie con elementi di ferro e tegole.

Al consolidamento dei frammenti di affresco conservati in situ si procede con la realizzazione di "lacertini" in stucco o con bendaggi di cemento e assicurandoli alle murature con grappe di ferro, nell'ambiente ipogeo di soggiorno (81) della Casa dell'Albergo (III, 1, 12).

Le lacune degli affreschi vengono reintegrate con una malta a fondo chiaro, o con stucco a base di lapillo o cemento, sulla cui base talora può essere delineata la sinopia della sintassi decorativa, come nella Casa dell'Erma di bronzo ${ }^{44}$.

Frequente e capillarmente diffusa è la pratica che prevede per le decorazioni parietali la sequenza di spolveratura, raschiatura dal salnitro, spalmatura con cera e benzina o, a partire dagli anni Cinquanta del Novecento, con plasticoat, e per gli stucchi, invece, il trattamento con formalina.

Le decorazioni pavimentali, invece, sono reintegrate con un cementizio di lapillo e malta e trattate con olio e petrolio.

Come dimostrato in modo esemplare da E. Sorbo per il caso di studio delle Terme Centrali, un approccio integrato, che coniughi il riesame delle fonti d'archivio ad un accurato esame dell'evidenza materiale ancora oggi in situ, apre la strada alla riscoperta delle strutture antiche, al di là e al di sotto degli interventi post-scavo e alle superfetazioni frutto della loro seconda vita ${ }^{45}$.

Per l'insula III, questa lettura contestuale, fra strutture in situ, fonti scritte e fonti iconografiche, documenti d'archivio e letteratura scientifica, ha consentito di realizzare un vero e proprio scavo stratigrafico nella storia conservativa dell'insula

44 Maiuri, 1958, p. 22.

45 Sorbo, 2014. Per un corpus degli interventi museografici di A. Maiuri ad Ercolano, Camardo, Notomista, 2017. 
e delle sue componenti e di gettare basi più solide per la comprensione della sua storia antica.

\section{Antonella Coralini}

Professore di Archeologia Classica

Responsabile Scientifico

del Programma Vesuviana

Alma Mater Studiorum

Università di Bologna

Dipartimento di Storia Culture Civiltà

Piazza S. Giovanni in Monte, 2

40124 Bologna $(\mathrm{I})$ 